\title{
Sex differences in the late first trimester human placenta transcriptome
}

Tania L. Gonzalez ${ }^{1}$, Tianyanxin Sun ${ }^{1}$, Alexander F. Koeppel², Bora Lee ${ }^{1}$, Erica T. Wang ${ }^{1,3}$, Charles R. Farber ${ }^{2}$, Stephen S. Rich ${ }^{2}$, Lauren W. Sundheimer ${ }^{1,3}$, Rae A. Buttle ${ }^{1,4}$, Yii-Der Ida Chen ${ }^{5}$, Jerome I. Rotter ${ }^{5}$, Stephen D. Turner ${ }^{2}$, John Williams $\mathrm{II}^{1,4}$, Mark O. Goodarzi ${ }^{1,6}$ and Margareta D. Pisarska ${ }^{1,3^{*}}$

\begin{abstract}
Background: Development of the placenta during the late first trimester is critical to ensure normal growth and development of the fetus. Developmental differences in this window such as sex-specific variation are implicated in later placental disease states, yet gene expression at this time is poorly understood.

Methods: RNA-sequencing was performed to characterize the transcriptome of 39 first trimester human placentas using chorionic villi following genetic testing (17 females, 22 males). Gene enrichment analysis was performed to find enriched canonical pathways and gene ontologies in the first trimester. DESeq2 was used to find sexually dimorphic gene expression. Patient demographics were analyzed for sex differences in fetal weight at time of chorionic villus sampling and birth.

Results: RNA-sequencing analyses detected 14,250 expressed genes, with chromosome 19 contributing the greatest proportion (973/2852, 34.1\% of chromosome 19 genes) and $Y$ chromosome contributing the least $(16 / 568,2.8 \%)$. Several placenta-enriched genes as well as histone-coding genes were identified to be unique to the first trimester and common to both sexes. Further, we identified 58 genes with significantly different expression between males and females: 25 X-linked, 15 Y-linked, and 18 autosomal genes. Genes that escape $X$ inactivation were highly represented (59.1\%) among $X$-linked genes upregulated in females. Many genes differentially expressed by sex consisted of $X / Y$ gene pairs, suggesting that dosage compensation plays a role in sex differences. These $X / Y$ pairs had roles in parallel, ancient canonical pathways important for eukaryotic cell growth and survival: chromatin modification, transcription, splicing, and translation.

Conclusions: This study is the first characterization of the late first trimester placenta transcriptome, highlighting similarities and differences among the sexes in ongoing human pregnancies resulting in live births. Sexual dimorphism may contribute to pregnancy outcomes, including fetal growth and birth weight, which was seen in our cohort, with males significantly heavier than females at birth. This transcriptome provides a basis for development of early diagnostic tests of placental function that can indicate overall pregnancy heath, fetal-maternal health, and long-term adult health.
\end{abstract}

Keywords: Sex differences, First trimester placenta, Chorionic villus sampling, Pregnancy, RNA-sequencing

\footnotetext{
* Correspondence: Margareta.Pisarska@cshs.org

${ }^{1}$ Department of Obstetrics and Gynecology, Division of Reproductive

Endocrinology and Infertility, Cedars-Sinai Medical Center, Los Angeles, CA, USA

${ }^{3}$ Division of Reproductive Endocrinology and Infertility, UCLA David Geffen

School of Medicine, Los Angeles, CA, USA

Full list of author information is available at the end of the article
} 


\section{Background}

In humans and other placental mammals, the fertilized egg gives rise to both the fetus and the placenta. Placentation in the first trimester can impact fetal growth, and abnormal placentation can lead to more pronounced effects complicating pregnancy including intrauterine growth restriction (IUGR) which results in very low birth weight infants, a sexually dimorphic outcome [1-4]. The process of placentation occurs throughout the first trimester of pregnancy, whereby the outer cells of the blastocyst (the trophoblast cells) invade the maternal tissue and develop into the placenta. It is a highly regulated state of active cell proliferation, cell migration, and cell differentiation [5]. To sustain this growth, placentation is in a high state of transcriptional activity, as evidenced by its marked hypomethylated state [5-9]. Placentation requires multiple factors, including maternal immune tolerance, various growth factors, fetal-maternal communication via chemical signaling, and a receptive maternal decidua that allows extravillous trophoblast cells to invade the maternal circulatory system and access maternal nutrients throughout pregnancy [5, 10-13].

Fetal growth, development, and well-being is impacted by fetal sex [14, 15]. Females are slightly smaller as early as $8-12$ weeks and continue to be smaller throughout gestation [16, 17]. Fetal survival rates are also sexually dimorphic in early pregnancy. Male fetuses have a 2.00 2.50-fold higher risk for spontaneous abortions in the first trimester and 1.25-fold higher risk before 23 weeks (midsecond trimester) [18]. Despite studies describing the placental transcriptome at term and differences attributed to fetal sex [19-21], there are no studies looking at the first trimester placenta, which is a critical time during development that may contribute to sexually dimorphic outcomes and is a time when pregnancy complications likely manifest $[7,22]$. In addition, it is during this time that origins of common adult diseases such as hypertension, coronary heart disease, type 2 diabetes, and depression may develop, which are also sexually dimorphic [23, 24].

Thus, our goal is to identify the late first trimester placenta transcriptome and identify gene expression differences due to fetal sex. This work provides a valuable reference of the late first trimester placental transcriptome at the earliest time point of placental development that can be studied in ongoing human pregnancies. It can be used to develop a sex-specific placenta atlas unique to humans, important because the human placenta has unique features not found in animals models [25]. In addition, findings from these studies may be the initial step in identifying potential mechanisms in placentation that lead to sexually dimorphic outcomes in fetal growth and development as well as long-term adult health, and can ultimately be used to develop noninvasive diagnostic testing of fetal well-being [26].

\section{Methods}

\section{Chorionic villi sample procurement}

First trimester placenta was obtained from discarded chorionic villi at 10.5-13.5 weeks from chorionic villus sampling (CVS) used for genetic testing, with informed written consent. All protocols were performed in accordance with the institutional review board's guidelines at the CedarsSinai Medical Center under IRB Protocols Pro00006806 (Prenatal repository) and Pro00008600 (differential gene expression of early placenta). Remaining CVS tissue that was not used for genetic testing was cleaned to separate maternal-derived decidua tissue and fetal-derived chorionic villi. Tissue samples (5-15 mg) were kept on ice and submerged in $250 \mu \mathrm{l}$ RNAlater RNA Stabilization Reagent (QIAGEN, Hilden, Germany) within $30 \mathrm{~min}$, then stored at $-80^{\circ} \mathrm{C}$ in Cedar-Sinai Medical Center's Prenatal Repository until further processing. All subjects were Caucasian, spontaneous singleton conceptions that resulted in a live birth. There were 17 females and 22 males. All karyotypes were either $46, \mathrm{XX}$ or $46, \mathrm{XY}$.

\section{Subject demographics analysis}

Subject demographics including maternal age, race, ethnicity, pre-pregnancy BMI, as well as fetal gestational age at CVS, crown-rump length (CRL), karyotype analysis, gestational age at delivery, and birthweight were analyzed for $n=39$ subjects. Dating by last menstrual period, when available, and early initial ultrasonography was completed using crown-rump length (CRL) measurements to confirm gestational age. Gestational age and CRL measured at the time of CVS were used for analysis. $t$ test was used for continuous variables and Fisher's exact test was used for categorical variables. Eight samples lacked complete clinical data for birthweight, gestational age, or crown-rump length. Omitting those samples (leaving $n=31$ ), the results were unchanged.

\section{RNA extraction from chorionic villi tissue}

CVS were processed as previously described [27]. Briefly, CVS tissue was thawed on ice with $600 \mu \mathrm{l}$ of RTL Plus lysis buffer (QIAGEN) and 1\% $\beta$-mercaptoethanol added to each sample. Cells were homogenized by passing at least 10 times through single-use needles with decreasing gauge (22G, 25G then $27 \mathrm{G}$ ) attached to a $1 \mathrm{~mL}$ sterile, RNase-free syringe. The homogenates were loaded onto AllPrep spin columns and the remainder of the protocol was performed following manufacturer instructions. DNA and RNA were extracted using the commercial AllPrep DNA/RNA Mini Kit (QIAGEN) for RNA-seq. RNA was eluted with $30 \mu \mathrm{l}$ of the kit-provided RNase-free water at room temperature, then the elution was passed through the column a second time to increase RNA yield. DNA was eluted with $100 \mu \mathrm{l}$ of the kit-provided EB buffer warmed to $70{ }^{\circ} \mathrm{C}$. Equal numbers of male and female 
samples were processed each time to reduce batch effects. The average RNA integrity number (RIN) score for RNA samples was 7.70 .

\section{RNA-sequencing and statistical analysis}

RNA-Seq libraries were constructed from $200 \mathrm{ng}$ of total RNA using Illumina TruSeq Stranded Total RNA with Ribo-Zero Gold sample prep kits (Illumina, Carlsbad, CA, USA). Constructed libraries contained RNAs $>200 \mathrm{nt}$ (both unpolyadenylated and polyadenylated) and were depleted of cytoplasmic and mitochondrial rRNAs. An average of 22.53 million $2 \times 75$ bp paired-end reads were generated for each sample on an Illumina NextSeq 500. Reads were aligned to the human reference genome (build GRCh38 with Ensembl release version 87 and UCSC release 24) using STAR [28] and we counted reads mapping to Ensembl genes using the featureCounts software in the Subread package [29]. We then used the DESeq2 Bioconductor package [30, 31] in the $\mathrm{R}$ statistical computing environment [32] to normalize count data, estimate dispersion, and fit a negative binomial model for each gene. The Benjamini-Hochberg false discovery rate (FDR) procedure was used to estimate the adjusted $P$ values for Ensembl gene IDs. Significant genes were defined as below 5\% FDR.

\section{Quality control to verify sample identity}

To ensure samples did not contain maternal contamination, RNA sequencing results were screened for deciduaspecific genes (SCARA5, PIGF, SLPI) and samples excluded if any decidua-specific genes were expressed. Fetal sex, identified on cytogenetic analysis, was also verified after RNA-sequencing using the individual baseMean and fragments per kilobase per million mapped fragments (FPKM) levels of XIST (a female-specific gene) and highly expressed $\mathrm{Y}$ chromosome genes (DDX3Y, $K D M 5 D, U T Y$, and $Z F Y$ ).

\section{Expression threshold selection}

In order to identify the transcription profile of the late first trimester human placenta of ongoing pregnancies, an expression threshold of FPKM > 1.281 was selected. After DESeq2 analysis, the mean FPKM from female samples was found for all Y-linked genes. The maximum value was 1.2808 for CD24P4, an outlier 13 standard deviations higher than the next value, 0.3968 for PSMA6P1. Signal for CD24P4 was likely sequencing noise from $C D 24$, a highly expressed chromosome 6 gene with $99.43 \%$ identity to CD24P4 (BLASTN $E$ value $=2.0 \times 10^{-90}$ ) found using the BLAST tool from the National Center for Biotechnology Information (NCBI) [33]. The higher threshold was selected to ensure a reliable transcriptome atlas. Genes on all chromosomes were considered expressed if they reached mean FPKM > 1.281 in females, males, or both.

\section{Pathway and gene enrichment analysis}

To investigate the biological significance of our gene subset, data were analyzed with Ingenuity Pathways Analysis (IPA) software (QIAGEN, Redwood City, CA, USA, www.qiagenbioinformatics.com/IPA), as previously described [34, 35]. Genes were partitioned into quartiles after sorting by maximum average FPKM (male or female). Gene groups were analyzed for enrichment in gene ontology, canonical pathways, and upstream regulators. A right-tailed Fisher's exact test was used to calculate $P$ values of enriched categories. The chromosome location of upstream regulators was found by cross-referencing gene names from IPA with the $\mathrm{db} 2 \mathrm{db}$ tool from biological DataBase network (Frederick National Laboratory for Cancer Research, https://biodbnet-abcc.ncifcrf.gov/db/ db2db.php) [36]. The top $25 \%$ of expressed genes were input into IPA for upstream analysis and 98.0\% (3493 of 3563) were accepted. Upstream regulators were ranked by most significant $P$ value (Fisher's exact test). Full names and descriptions were pulled from the National Institute of Health NCBI databases (Gene, PubChem) and miRbase: the microRNA database [33, 37]. For each upstream regulator, the percent of accepted input genes that it affects is shown as a percentage (e.g., $195 / 3493 \times 100 \%$ for Dglucose) in column "\% Genes Regulated."

\section{Identification of placental specific or enriched transcripts}

In order to identify highly expressed genes with placenta tissue-specificity, we performed an in silico analysis of tissue specificity on genes with FPKM $>128$. Proteincoding genes were ranked by highest FPKM value across all samples in the first trimester. We referenced the expressed sequence tag (EST) profile breakdown by healthy body sites (typically 45 sites) of protein-coding genes using the NCBI UniGene database (https:// www.ncbi.nlm.nih.gov/unigene) accessed 15 Aug 2017 [33]. To determine placenta enrichment, the transcripts per million (TPM) of protein-coding genes in different normal body sites were compared. Genes with no placenta expression in UniGene $(\mathrm{TPM}=0)$ were validated to be FPKM $<2$ in term placenta using RNA-seq data from NCBI Gene Expression Omnibus (GEO) database, accession GSE73016 [38].

\section{Venn diagram of gene ontology for sexually dimorphic genes}

The Venn diagram for differentially expressed genes (DEGs) was created with modifications from Bellott et al. [39]. Additional functional categories were added in an effort to maximize the number of sexually dimorphic genes represented. The NCBI Gene database (https://www.ncbi.nlm. nih.gov/gene) and UniProt [40] were used for an initial ontology scan then PubMed was cross-referenced to verify gene ontology (https://www.ncbi.nlm.nih.gov/ 
pubmed/). Almost all DEGs (43/58; 74.2\%) had known or predicted function.

\section{Overview of $\mathrm{X}$ chromosome inactivation status and $\mathrm{X} / \mathrm{Y}$ gene homology}

The human genome annotation file (GRCh38) was downloaded from Ensembl [41]. The annotations for the 25 differentially expressed X-linked genes (FDR <0.05) were extracted from the human genome annotation file and input to NCBI Genome Decoration Page (https:// www.ncbi.nlm.nih.gov/genome/tools/gdp) to generate the ideogram of the $\mathrm{X}$ chromosome showing the locations of these genes. The consensus calls for $\mathrm{X}$ inactivation status were adapted from Balaton et al. [42], which compiled a comprehensive list of $\mathrm{X}$ inactivation statuses from several studies. Genes that were reported to be subject to $\mathrm{X}$ inactivation in most or all of these studies were categorized as $\mathrm{X}$ inactivated genes (color-coded red). Genes that were reported to be escaping $\mathrm{X}$ inactivation in most or all of these studies were categorized here as escaping genes (color-coded blue), including one gene (CHM) that had an even split of X chromosome inactivation (XCI) status calls between studies. On the human X chromosome, 25\% of $\mathrm{X}$-linked genes located outside of pseudo-autosomal regions have $Y$ counterparts [42], which are either functional ("Y homologs") or non-functional ("Y pseudogenes") [39, 43]. Table 7 listed all $X / Y$ gene pairs that contain functional $Y$ homologs, but not non-functional $\mathrm{Y}$ pseudogenes. All X-linked genes listed in Table 7 are protein-coding genes.

\section{Results}

Identification of a transcriptome signature from late first trimester human placenta

To identify the transcriptional profile of the late first trimester human placenta, RNA sequencing was performed on chorionic villi from 39 singleton pregnancies (17 female and 22 male samples). RNA-sequencing resulted in approximately 22.53 million $2 \times 75$ bp pairended reads per sample. Genes were annotated using human reference build GRCh38. Out of 57,543 human genes, a total of 47,778 genes had non-zero baseMean values indicating possible detection in chorionic villi. Principal components analysis showed clusters separated by sex (Additional file 1A). To determine whether each gene was expressed or not in chorionic villi regardless of sex, the FPKM values were used to identify a normative first trimester placental signature. An FPKM > 1.281 threshold was selected to remove sequencing noise, higher than the typical arbitrary threshold of FPKM $>1$. This higher threshold was selected to remove all background sequencing noise which produced low levels of $\mathrm{Y}$ chromosome signal from cytogenetically confirmed female samples (no Y chromosome). A total of 14,250 genes were determined to comprise the normative firsttrimester placenta transcriptome: 13,319 present in both males and females, and the remaining 931 genes above the expression threshold in one sex but not the other. The $\log _{2}$ FPKM of expressed genes, as an average of all samples, ranged from -3.7356 to 16.3245 (Fig. 1a).

The chromosomal distribution of the expressed genes (protein-coding, long non-coding, pseudogenes, and short non-coding) are presented in Fig. 1b. The highest number of expressed genes is on chromosome 1 followed by one of the shortest chromosomes, chromosome 19 (Fig. 1b). Since gene distribution is not proportional to chromosome size, we looked at the proportion of expressed genes in the placenta as a percentage of total genes for each chromosome using human genome assembly GRCh38.p7 [41]. Even after controlling for gene distribution, 34.1\% $(973 / 2852)$ of genes on chromosome 19 were expressed, the highest of any other chromosome. The Y chromosome had the lowest proportion of expressed genes with only $2.8 \%$ (16 genes) of all $568 \mathrm{Y}$ chromosome genes annotated in human genome assembly GRCh38.p7. Of these $16 \mathrm{Y}$ linked genes, there were 8 protein-coding, 4 long noncoding, and 4 pseudogenes.

\section{Expressed genes in the late first trimester human placenta}

The biotypes of all expressed transcripts of the normative late first trimester placenta transcriptome consist of $77.0 \%$ protein-coding genes, $9.8 \%$ long non-coding genes, $6.5 \%$ pseudogenes, and $6.7 \%$ short non-coding genes (Fig. 1c). Expressed genes were sorted by highest-to-lowest FPKM values using the female average or male average for each gene (whichever was higher) and separated into quartiles of approximately 3562 genes each. This method best represented the high expression of sex-specific genes such as Y-linked genes absent in females, which would otherwise drop from the highest quartile to the lowest quartile if the average FPKM of all 39 samples were used. The biotype distributions of the top two quartiles were similar in the first trimester placenta. Within the highest quartile (top 25\% expressed genes), 94.4\% (3364) were protein-coding genes, $1.3 \%$ (48) were long non-coding, $1.1 \%$ (40) were pseudogenes, and $3.1 \%$ (111) were short non-coding (Fig. 1c, Additional file 2). Of the short non-coding genes in the highest quartile, $32.4 \%$ (36 of 111) were microRNAencoding genes, of which $66.7 \%$ (24 of 36 ) were located on chromosome 19. Within the second highest quartile (51$75 \%$ of sorted genes), $90.8 \%$ (3234) were protein-coding, $3.1 \%$ (111) were long non-coding, $2.7 \%$ (97) were pseudogenes, and $3.4 \%$ (120) were short non-coding genes (Fig. 1c). The proportion of non-coding genes only reached $>10 \%$ in the lower two quartiles (Fig. 1c).

Ingenuity Pathways Analysis (IPA) for gene enrichment analysis was performed for expressed genes in the 

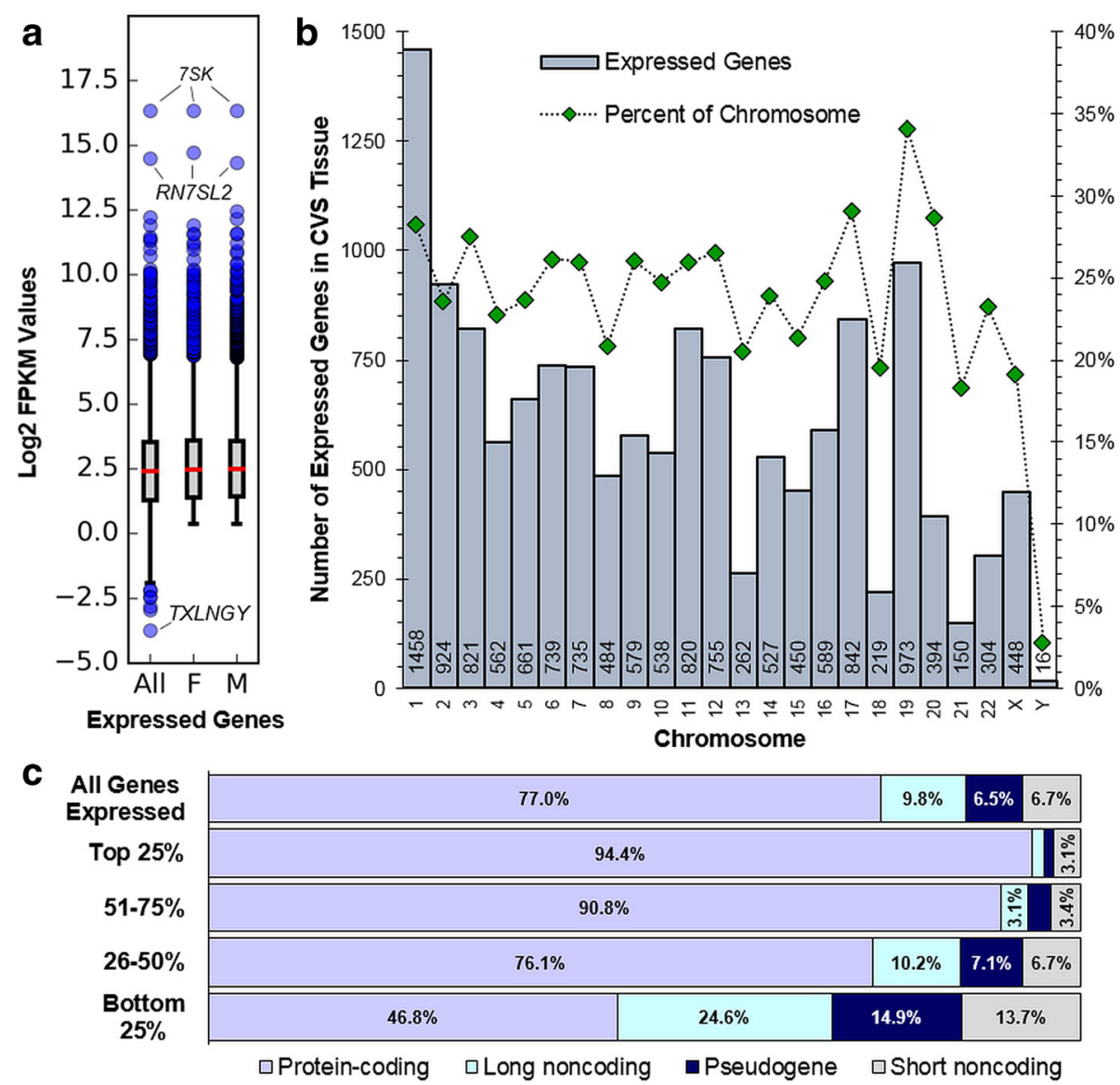

Fig. 1 Normative late first trimester placenta transcriptome. a Box and whisker plot showing $\log _{2}$ FPKM distribution of expressed genes. All: average $\log _{2}$ FPKM of 14,250 expressed genes over all 39 samples. F, M: all genes over the FPKM cutoff in either female or male samples, respectively. The whiskers range is the interquartile range $\pm 50 \%$, with outliers shown as blue circles. Median is red line. $\mathbf{b}$ Chromosome distribution of 14,250 genes expressed in chorionic villi, all biotypes (gray bars). Expressed genes are shows as a percentage of total genes in each chromosome (green diamonds). $\mathbf{c}$ Biotype categories of all expressed genes and each FPKM quartile are shown. The proportion of each biotype is shown as a percentage and labeled if $\geq 3 \%$

first trimester placenta, partitioned into quartiles (Table 1). In the most highly expressed quartile, the enriched canonical pathways were EIF2 signaling, regulation of eIF4 and p70S6K signaling, protein ubiquitination, mitochondrial dysfunction, and sirtuin signaling. These pathways are essential for protein synthesis, cell growth, and energy metabolism. Within the second highest quartile, the most enriched canonical pathways were related to hormonal signaling and growth regulation, including estrogen, JAK/ STAT, ceramide, insulin, and STAT3 signaling.

Among the top 25\% most expressed genes, the most enriched molecular and cellular functions were cell death and survival, protein synthesis, cellular development, cell growth and proliferation, and cellular movement (Table 2). The top five enriched categories for physiological systems development and function were organismal survival, cardiovascular systems development and function, connective tissue development and function, tissue development, and organismal development (Table 2). Upstream analysis in
IPA identified the top 40 gene regulators upstream of the top 25\% expressed genes (Additional file 3). Most upstream regulators were associated with either essential cell regulation, cell growth, or hormonal signaling. Transcriptional regulators such as MYC $\left(P=1.70 \times 10^{-70}\right)$, p53 $\left(P=1.63 \times 10^{-65}\right)$, MYCN $\left(P=3.17 \times 10^{-63}\right)$, and HNF4A $\left(P=2.10 \times 10^{-58}\right)$ were among the most significant upstream regulators (Additional file 3). Regulators involved in cell growth include rapamycin-insensitive companion of mTOR (RICTOR), transforming growth factor beta 1 (TGFB1), D-glucose, epidermal growth factor receptor $(E G F R)$, as well as epidermal growth factor $(E G F)$. In addition, hormones such as beta-estradiol, its receptor (ESR1), as well as the progesterone receptor $(P G R)$ were also significant upstream regulators. Together, these upstream regulators control essential cell regulation, cell growth, and hormonal signaling, consistent with the canonical pathways identified (Additional file 3). 
Table 1 Enriched canonical pathways for expressed genes in the late first trimester placenta, by FPKM quartiles

\begin{tabular}{|c|c|c|c|}
\hline Input & Top canonical pathways & $P$ value $^{\mathrm{a}}$ & \# genes \\
\hline \multirow[t]{5}{*}{ Top $25 \%$ of genes } & EIF2 signaling & $8.25 \mathrm{E}-55$ & 136 \\
\hline & Regulation of elF4 and p70S6K signaling & $7.88 \mathrm{E}-26$ & 81 \\
\hline & Protein ubiquitination pathway & $5.90 \mathrm{E}-25$ & 111 \\
\hline & Mitochondrial dysfunction & 1.07E-22 & 81 \\
\hline & Sirtuin signaling pathway & 5.43E-20 & 109 \\
\hline \multirow[t]{5}{*}{$51-75 \%$ quartile } & Estrogen receptor signaling & 2.12E-10 & 48 \\
\hline & JAKSSTAT signaling & 9.83E-08 & 32 \\
\hline & Ceramide signaling & $1.80 \mathrm{E}-07$ & 34 \\
\hline & Insulin receptor signaling & 2.18E-07 & 45 \\
\hline & STAT3 pathway & 2.57E-07 & 29 \\
\hline \multirow[t]{5}{*}{$26-50 \%$ quartile } & Melanocyte development and pigmentation signaling & 7.85E-04 & 24 \\
\hline & NGF signaling & 7.96E-04 & 28 \\
\hline & ERK/MAPK signaling & $1.59 \mathrm{E}-03$ & 40 \\
\hline & HGF signaling & 1.72E-03 & 26 \\
\hline & Acyl-CoA hydrolysis & $1.76 \mathrm{E}-03$ & 6 \\
\hline \multirow[t]{5}{*}{ 0-25\% quartile } & PI3K signaling in B lymphocytes & $9.40 \mathrm{E}-04$ & 21 \\
\hline & CDP-diacylglycerol biosynthesis I & $1.71 \mathrm{E}-03$ & 7 \\
\hline & Wnt/Ca ${ }^{2+}$ pathway & 2.81E-03 & 12 \\
\hline & Phosphatidylglycerol biosynthesis II (non-plastidic) & 2.83E-03 & 7 \\
\hline & D-myo-inositol-5-phosphate metabolism & $3.20 \mathrm{E}-03$ & 23 \\
\hline
\end{tabular}

${ }^{\mathrm{a}}$ Fisher's exact test

In order to identify highly expressed genes with placentaenriched tissue expression, we selected protein-coding genes with FPKM $>128$, two orders of magnitude above the expression threshold followed by an in silico assessment that compared them to EST profiles of healthy human tissue in the NCBI UniGene database (http://www.ncbi.nlm.nih.gov/unigene/) (Table 3). There were 175 protein-coding genes with FPKM $>128$ in the first trimester placenta, with the highest expression from KISS1 (metastasis-suppressor), CGA (glycoprotein hormones alpha subunit), TFPI2 (tissue factor pathway inhibitor 2), $\mathrm{CSH} 1$ and $\mathrm{CSH} 2$ (chorionic somatomammotropin hormones), CGB8 and CGB5 (chorionic gonadotropin beta subunits), SPP1 (secreted phosphoprotein 1), TMSB10 (thymosin beta 10), and EEF1A1 (eukaryotic translation elongation factor 1 alpha 1). Of these 175 protein-coding genes, $35(20 \%)$ were known to

Table 2 Enriched gene ontology for top 25\% of expressed genes

\begin{tabular}{|c|c|c|}
\hline & $P$ value range ${ }^{a}$ & $\overline{\# \text { \# genes }}$ \\
\hline \multicolumn{3}{|l|}{ Molecular and cellular function } \\
\hline Cell death and survival & 7.97E-11 to $2.76 \mathrm{E}-96$ & 1356 \\
\hline Protein synthesis & $3.01 \mathrm{E}-11$ to $8.66 \mathrm{E}-70$ & 598 \\
\hline Cellular development & $3.61 \mathrm{E}-11$ to $1.31 \mathrm{E}-57$ & 999 \\
\hline Cellular growth and proliferation & $3.61 \mathrm{E}-11$ to $1.31 \mathrm{E}-57$ & 953 \\
\hline Cellular movement & $1.05 \mathrm{E}-10$ to $6.29 \mathrm{E}-53$ & 892 \\
\hline \multicolumn{3}{|l|}{ Physiological systems development and function } \\
\hline Organismal survival & $1.31 \mathrm{E}-15$ to $1.52 \mathrm{E}-63$ & 958 \\
\hline Cardiovascular system development and function & $9.45 \mathrm{E}-11$ to $1.20 \mathrm{E}-31$ & 576 \\
\hline Connective tissue development and function & $8.86 \mathrm{E}-11$ to $4.80 \mathrm{E}-27$ & 579 \\
\hline Tissue development & $7.01 \mathrm{E}-11$ to $4.80 \mathrm{E}-27$ & 747 \\
\hline Organismal development & $9.45 \mathrm{E}-11$ to $3.54 \mathrm{E}-23$ & 1008 \\
\hline
\end{tabular}

${ }^{a}$ Fisher's exact test for sub-categories from IPA shown as $P$ value range 
Table 3 Highest expressed protein-coding genes in late first trimester placenta

\begin{tabular}{|c|c|c|c|c|c|c|c|}
\hline$\#$ & $\begin{array}{l}\text { Gene } \\
\text { symbol }\end{array}$ & Chr & Description & $\begin{array}{l}\text { TPM highest } \\
\text { in placenta? }\end{array}$ & $\begin{array}{l}\text { Placenta } \\
\text { specific? }^{\mathrm{b}}\end{array}$ & Where else expressed? ${ }^{\mathrm{b}}$ & $\begin{array}{l}\text { Upregulated in } \\
\text { first vs term }{ }^{c}\end{array}$ \\
\hline 1 & KISS1 & 1 & KiSS-1 metastasis-suppressor & +++ & N & & Term \\
\hline 2 & CGA & 6 & Glycoprotein hormones, alpha polypeptide & $\mathrm{N}$ & N & & \\
\hline 3 & TFPI2 & 7 & Tissue factor pathway inhibitor 2 & $\mathrm{~N}$ & N & & \\
\hline 4 & $\mathrm{CSH} 1$ & 17 & Chorionic somatomammotropin hormone 1 & ++ & N & & \\
\hline 5 & $\mathrm{CSH} 2$ & 17 & Chorionic somatomammotropin hormone 2 & + & N & & \\
\hline 6 & CGB8 & 19 & Chorionic gonadotropin beta subunit 8 & ++ & Almost & Embryonic tissue, skin & \\
\hline 7 & CGB5 & 19 & Chorionic gonadotropin beta subunit 5 & ++ & Almost & $\begin{array}{l}\text { Embryonic tissue, testis, } \\
\text { heart, skin }\end{array}$ & \\
\hline 8 & SPP1 & 4 & Secreted phosphoprotein 1 & $\mathrm{~N}$ & N & & \\
\hline 9 & TMSB10 & 2 & Thymosin beta 10 & N & N & & \\
\hline 10 & EEF1A1 & 6 & Eukaryotic translation elongation factor 1 alpha 1 & N & N & & \\
\hline 11 & PEG10 & 7 & Paternally expressed 10 & $\mathrm{~N}$ & N & & \\
\hline 12 & FTL & 19 & Ferritin light chain & $\mathrm{N}$ & N & & \\
\hline 13 & CGB3 & 19 & Chorionic gonadotropin beta subunit 3 & ++ & Almost & $\begin{array}{l}\text { Embryonic tissue, connective } \\
\text { tissue, testis, ovary }\end{array}$ & \\
\hline 14 & RPS12 & 6 & Ribosomal protein S12 & N & N & & \\
\hline 15 & PSG3 & 19 & Pregnancy specific beta-1-glycoprotein 3 & +++ & Almost & $\begin{array}{l}\text { Vascular, testis, muscle, } \\
\text { kidney, intestine, skin }\end{array}$ & Term \\
\hline 16 & ACTB & 7 & Actin beta & N & N & & \\
\hline 17 & IFI6 & 1 & Interferon alpha inducible protein 6 & $\mathrm{~N}$ & N & & \\
\hline 18 & COL3A1 & 2 & Collagen type III alpha 1 chain & N & N & & \\
\hline 19 & GDF15 & 19 & Growth differentiation factor 15 & ++ & N & & Term \\
\hline 20 & PAGE4 & $x$ & PAGE family member 4 & + & N & Prostate, heart & \\
\hline 21 & RPS27 & 1 & Ribosomal protein S27 & $\mathrm{N}$ & N & & \\
\hline 22 & ACTG1 & 17 & Actin gamma 1 & $\mathrm{~N}$ & N & & \\
\hline 23 & FN1 & 2 & Fibronectin 1 & $\mathrm{~N}$ & N & & \\
\hline 24 & RPL13A & 19 & Ribosomal protein L13a & $\mathrm{N}$ & N & & \\
\hline 25 & $\mathrm{HIST} 1 \mathrm{H} 1 \mathrm{C}$ & 6 & Histone cluster $1 \mathrm{H} 1$ family member $\mathrm{c}$ & N & N & & \\
\hline 26 & RPS11 & 19 & Ribosomal protein S11 & $\mathrm{N}$ & N & & \\
\hline 27 & S100A11 & 1 & S100 calcium binding protein A11 & N & N & & \\
\hline 28 & HIST1H2BK & 6 & Histone cluster 1 H2B family member $\mathrm{k}$ & $\mathrm{N}$ & N & & \\
\hline 29 & HIST1H3B & 6 & Histone cluster $1 \mathrm{H} 3$ family member b & No value & Almost & Thymus, liver, kidney, testis & \\
\hline 30 & HIST1H1E & 6 & Histone cluster $1 \mathrm{H} 1$ family member e & No value & Almost & Mouth, kidney, brain & \\
\hline 31 & PSAP & 10 & Prosaposin & N & N & & \\
\hline 32 & EEF2 & 19 & Eukaryotic translation elongation factor 2 & $\mathrm{~N}$ & N & & \\
\hline 33 & RPS18 & 6 & Ribosomal protein S18 & $\mathrm{N}$ & N & & \\
\hline 34 & RPL10A & 6 & Ribosomal protein L10a & $\mathrm{N}$ & N & & \\
\hline 35 & CALR & 19 & Calreticulin & N & N & & \\
\hline 36 & HIST1H1B & 6 & Histone cluster $1 \mathrm{H} 1$ family member $\mathrm{b}$ & No value & Almost & Bladder, intestine, skin & \\
\hline 37 & TGM2 & 20 & Transglutaminase 2 & N & N & & \\
\hline 38 & RPL7A & 9 & Ribosomal protein L7a & $\mathrm{N}$ & N & & \\
\hline 39 & SAT1 & $x$ & Spermidine/spermine N1-acetyltransferase 1 & N & N & & \\
\hline 40 & XAGE3 & $x$ & $\mathrm{X}$ antigen family member 3 & + & Yes & & \\
\hline
\end{tabular}


Table 3 Highest expressed protein-coding genes in late first trimester placenta (Continued)

\begin{tabular}{|c|c|c|c|c|c|c|c|}
\hline \# & $\begin{array}{l}\text { Gene } \\
\text { symbol }\end{array}$ & Chr & Description & $\begin{array}{l}\text { TPM highest } \\
\text { in placenta? }\end{array}$ & $\begin{array}{l}\text { Placenta } \\
\text { specific? }^{\mathrm{b}}\end{array}$ & Where else expressed? ${ }^{\mathrm{b}}$ & $\begin{array}{l}\text { Upregulated in } \\
\text { first vs term }{ }^{c}\end{array}$ \\
\hline$\overline{41}$ & HIST1H4D & 6 & Histone cluster $1 \mathrm{H} 4$ family member $\mathrm{d}$ & No value & Almost & Adipose tissue, vascular & \\
\hline 42 & HSPA8 & 11 & Heat shock protein family A ( Hsp70) member 8 & $N$ & N & & \\
\hline 43 & PSG4 & 19 & Pregnancy specific beta-1-glycoprotein 4 & ++ & N & & Term \\
\hline 44 & GAPDH & 12 & Glyceraldehyde-3-phosphate dehydrogenase & $\mathrm{N}$ & N & & \\
\hline 45 & $\mathrm{EBI} 3$ & 19 & Epstein-Barr virus induced 3 & No value* & N & & Term \\
\hline 46 & RPS4X & $x$ & Ribosomal protein $\mathrm{S} 4, \mathrm{X}$-linked & N & N & & \\
\hline 47 & RPS6 & 9 & Ribosomal protein $\mathbf{S 6}$ & N & N & & \\
\hline 48 & RPS8 & 1 & Ribosomal protein S8 & N & N & & \\
\hline 49 & EFEMP1 & 2 & $\begin{array}{l}\text { EGF containing fibulin-like extracellular matrix protein } \\
1\end{array}$ & N & N & & \\
\hline 50 & RPLPO & 12 & Ribosomal protein lateral stalk subunit PO & N & N & & \\
\hline 51 & ADAM12 & 10 & ADAM metallopeptidase domain 12 & ++ & N & & Term \\
\hline 52 & S100A9 & 1 & S100 calcium binding protein A9 & N & N & & Term \\
\hline 53 & CYP19A1 & 15 & Cytochrome P450 family 19 subfamily A member 1 & +++ & N & & Term \\
\hline 54 & COL4A1 & 13 & Collagen type IV alpha 1 chain & N & N & & \\
\hline 55 & ANXA5 & 4 & Annexin A5 & $N$ & N & & \\
\hline 56 & RPL19 & 17 & Ribosomal protein L19 & $N$ & N & & \\
\hline 57 & KRT18 & 12 & Keratin 18 & $N$ & N & & \\
\hline 58 & XAGE2 & $x$ & $\mathrm{X}$ antigen family member 2 & ++ & Almost & Heart, uterus & \\
\hline 59 & HSP90AB1 & 6 & Heat shock protein 90 alpha family class B member 1 & N & N & & \\
\hline 60 & HIST1H4C & 6 & Histone cluster $1 \mathrm{H} 4$ family member c & No value & N & & \\
\hline 61 & IGF2 & 11 & Insulin-like growth factor 2 & $N$ & N & & \\
\hline 62 & HSD3B1 & 1 & $\begin{array}{l}\text { Hydroxy-delta-5-steroid dehydrogenase, } 3 \text { beta- and } \\
\text { steroid delta-isomerase } 1\end{array}$ & ++ & N & & \\
\hline 63 & EZR & 6 & Ezrin & $N$ & N & & \\
\hline 64 & SLC2A1 & 1 & Solute carrier family 2 member 1 & $N$ & N & & Term \\
\hline 65 & RPS2 & 16 & Ribosomal protein S2 & N & N & & \\
\hline 66 & FBLN1 & 22 & Fibulin 1 & + & N & & Term \\
\hline 67 & $\mathrm{~S} 100 \mathrm{P}$ & 4 & S100 calcium binding protein $P$ & N & N & & Term \\
\hline 68 & SDC1 & 2 & Syndecan 1 & $N$ & N & & Term \\
\hline 69 & HIST1H3F & 6 & Histone cluster $1 \mathrm{H} 3$ family member $\mathrm{f}$ & No value & Almost & $\begin{array}{l}\text { Liver, connective tissue, } \\
\text { skin }\end{array}$ & \\
\hline 70 & CTSL & 9 & Cathepsin L & N & N & & \\
\hline 71 & HIST1H4F & 6 & Histone cluster $1 \mathrm{H} 4$ family member $\mathrm{f}$ & No value & Almost & Blood, connective tissue & \\
\hline 72 & PSG1 & 19 & Pregnancy specific beta-1-glycoprotein 1 & +++ & N & & Term \\
\hline 73 & RPS25 & 11 & Ribosomal protein S25 & $\mathrm{N}$ & N & & \\
\hline 74 & PSG5 & 19 & Pregnancy specific beta-1-glycoprotein 5 & ++ & N & & \\
\hline 75 & PLAC4 & 21 & Placenta specific 4 & +++ & N & & \\
\hline 76 & RPL11 & 1 & Ribosomal protein L11 & N & N & & \\
\hline 77 & SPARC & 5 & Secreted protein acidic and cysteine rich & N & N & & \\
\hline 78 & KRT19 & 17 & Keratin 19 & $\mathrm{~N}$ & N & & \\
\hline 79 & PSG2 & 19 & Pregnancy specific beta-1-glycoprotein 2 & +++ & N & & \\
\hline 80 & RPL8 & 8 & Ribosomal protein L8 & N & N & & \\
\hline
\end{tabular}


Table 3 Highest expressed protein-coding genes in late first trimester placenta (Continued)

\begin{tabular}{|c|c|c|c|c|c|c|c|}
\hline$\#$ & $\begin{array}{l}\text { Gene } \\
\text { symbol }\end{array}$ & Chr & Description & $\begin{array}{l}\text { TPM highest } \\
\text { in placenta? }\end{array}$ & $\begin{array}{l}\text { Placenta } \\
\text { specific? }^{\mathrm{b}}\end{array}$ & Where else expressed? ${ }^{\mathrm{b}}$ & $\begin{array}{l}\text { Upregulated in } \\
\text { first vs term }{ }^{c}\end{array}$ \\
\hline 81 & HSP90B1 & 12 & Heat shock protein 90 beta family member 1 & $\mathrm{~N}$ & N & & \\
\hline 82 & GPC3 & $x$ & Glypican 3 & $\mathrm{~N}$ & N & & \\
\hline 83 & RPL7 & 8 & Ribosomal protein L7 & $\mathrm{N}$ & N & & \\
\hline 84 & PAPPA2 & 1 & Pappalysin 2 & ++ & N & & Term \\
\hline 85 & KRT8 & 12 & Keratin 8 & + & N & & \\
\hline 86 & RPL5 & 1 & Ribosomal protein $\mathrm{L} 5$ & $\mathrm{~N}$ & N & & \\
\hline 87 & $\mathrm{GH} 2$ & 17 & Growth hormone 2 & + & N & & \\
\hline 88 & MEST & 7 & Mesoderm specific transcript & $\mathrm{N}$ & N & & \\
\hline 89 & RPN2 & 20 & Ribophorin ॥ & $\mathrm{N}$ & N & & \\
\hline 90 & PRDX5 & 11 & Peroxiredoxin 5 & N & N & & \\
\hline 91 & GSTP1 & 11 & Glutathione S-transferase pi 1 & $\mathrm{~N}$ & N & & \\
\hline 92 & LDHB & 12 & Lactate dehydrogenase B & $\mathrm{N}$ & N & & \\
\hline 93 & HIST1H3C & 6 & Histone cluster $1 \mathrm{H} 3$ family member $\mathrm{c}$ & No value & Almost & Testis & \\
\hline 94 & VGLL1 & $x$ & Vestigial-like family member 1 & ++ & N & & \\
\hline 95 & RPL4 & 15 & Ribosomal protein $\llcorner 4$ & N & N & & \\
\hline 96 & ENO1 & 1 & Enolase 1 & $\mathrm{~N}$ & N & & First \\
\hline 97 & PAPPA & 9 & Pappalysin 1 & ++ & N & & Term \\
\hline 98 & COL4A2 & 13 & Collagen type IV alpha 2 chain & $\mathrm{N}$ & N & & \\
\hline 99 & RPS3A & 4 & Ribosomal protein S3A & $\mathrm{N}$ & N & & \\
\hline 100 & SLC40A1 & 2 & Solute carrier family 40 member 1 & $\mathrm{~N}$ & N & & \\
\hline 101 & TIMP3 & 22 & TIMP metallopeptidase inhibitor 3 & $\mathrm{~N}$ & N & & \\
\hline 102 & ANXA1 & 9 & Annexin A1 & $\mathrm{N}$ & N & & \\
\hline 103 & RPL35 & 9 & Ribosomal protein L35 & N & N & & \\
\hline 104 & TPT1 & 13 & Tumor protein, translationally controlled 1 & $\mathrm{~N}$ & N & & \\
\hline 105 & EPAS1 & 2 & Endothelial PAS domain protein 1 & N & N & & \\
\hline 106 & $\mathrm{HIST} 1 \mathrm{H} 2 \mathrm{BI}$ & 6 & Histone cluster $1 \mathrm{H} 2 \mathrm{~B}$ family member i & No value & Yes & $\begin{array}{l}\text { No value in any UniGene } \\
\text { body site }\end{array}$ & \\
\hline 107 & S100A6 & 1 & S100 calcium binding protein A6 & $\mathrm{N}$ & N & & \\
\hline 108 & RPL3 & 22 & Ribosomal protein L3 & $\mathrm{N}$ & N & & \\
\hline 109 & RPL12 & 9 & Ribosomal protein L12 & $\mathrm{N}$ & N & & \\
\hline 110 & DUSP9 & $x$ & Dual specificity phosphatase 9 & $\mathrm{~N}$ & N & & \\
\hline 111 & PSG9 & 19 & Pregnancy specific beta-1-lycoprotein 9 & +++ & Almost & $\begin{array}{l}\text { Vascular, spleen, eye, } \\
\text { mammary gland, lung, skin }\end{array}$ & Term \\
\hline 112 & $\mathrm{FOXO4}$ & $x$ & Forkhead box $\mathrm{O} 4$ & ++ & N & & \\
\hline 113 & PEG3 & 19 & Paternally expressed 3 & $\mathrm{~N}$ & N & & \\
\hline 114 & RPL41 & 12 & Ribosomal protein $L 41$ & $\mathrm{~N}$ & N & & \\
\hline 115 & FAM129B & 9 & Family with sequence similarity 129 member B & $\mathrm{N}$ & N & & \\
\hline 116 & PDIA3 & 15 & Protein disulfide isomerase family A member 3 & $\mathrm{~N}$ & N & & \\
\hline 117 & HIST1H4B & 6 & Histone cluster $1 \mathrm{H} 4$ family member b & No value & Almost & Lymph node, testis, liver & \\
\hline 118 & MYL12B & 18 & Myosin light chain 12B & $\mathrm{N}$ & N & & \\
\hline 119 & HIST1H1D & 6 & Histone cluster $1 \mathrm{H} 1$ family member $\mathrm{d}$ & No value & N & & \\
\hline 120 & SPINT1 & 15 & Serine peptidase inhibitor, Kunitz type 1 & + & N & & \\
\hline 121 & RPLP2 & 11 & Ribosomal protein lateral stalk subunit P2 & $\mathrm{N}$ & N & & \\
\hline
\end{tabular}


Table 3 Highest expressed protein-coding genes in late first trimester placenta (Continued)

\begin{tabular}{|c|c|c|c|c|c|c|c|}
\hline$\#$ & $\begin{array}{l}\text { Gene } \\
\text { symbol }\end{array}$ & Chr & Description & $\begin{array}{l}\text { TPM highest } \\
\text { in placenta? }^{\mathrm{a}}\end{array}$ & $\begin{array}{l}\text { Placenta } \\
\text { specific? }^{\mathrm{b}}\end{array}$ & Where else expressed? & $\begin{array}{l}\text { Upregulated in } \\
\text { first vs term }\end{array}$ \\
\hline 122 & FBN2 & 5 & Fibrillin 2 & + & $\mathrm{N}$ & & \\
\hline 123 & RPS27A & 2 & Ribosomal protein S27a & $N$ & N & & \\
\hline 124 & BSG & 19 & Basigin (Ok blood group) & N & N & & \\
\hline 125 & CTNNB1 & 3 & Catenin beta 1 & $N$ & N & & \\
\hline 126 & RPL21 & 13 & Ribosomal protein L21 & N & N & & \\
\hline 127 & COL6A2 & 21 & Collagen type VI alpha 2 chain & $\mathrm{N}$ & N & & First \\
\hline 128 & RASA1 & 5 & RAS p21 protein activator 1 & ++ & $\mathrm{N}$ & & \\
\hline 129 & ATP5B & 12 & $\begin{array}{l}\text { ATP synthase, } \mathrm{H}+\text { transporting, mitochondrial F1 } \\
\text { complex, beta polypeptide }\end{array}$ & N & N & & \\
\hline 130 & RPS20 & 8 & Ribosomal protein $\mathbf{S} 20$ & N & $\mathrm{N}$ & & \\
\hline 131 & RPS13 & 11 & Ribosomal protein S13 & $N$ & $\mathrm{~N}$ & & \\
\hline 132 & CANX & 5 & Calnexin & $N$ & N & & \\
\hline 133 & ISM2 & 14 & Isthmin 2 & +++ & N & & \\
\hline 134 & RPL9 & 4 & Ribosomal protein L9 & N & N & & \\
\hline 135 & HBG2 & 11 & Hemoglobin subunit gamma 2 & N & N & & \\
\hline 136 & RACK1 & 5 & Receptor for activated $\mathrm{C}$ kinase 1 & N & N & & \\
\hline 137 & LUM & 12 & Lumican & $\mathrm{N}$ & N & & \\
\hline 138 & NPM1 & 5 & Nucleophosmin & N & $\mathrm{N}$ & & \\
\hline 139 & $\mathrm{HIST} 1 \mathrm{H} 2 \mathrm{BO}$ & 6 & Histone cluster $1 \mathrm{H} 2 \mathrm{~B}$ family member $\mathrm{O}$ & No value* & Almost & Liver & \\
\hline 140 & PEBP1 & 12 & Phosphatidylethanolamine binding protein 1 & N & N & & \\
\hline 141 & RPL10 & $x$ & Ribosomal protein L10 & $\mathrm{N}$ & $\mathrm{N}$ & & \\
\hline 142 & $\mathrm{P} 4 \mathrm{HB}$ & 17 & Prolyl 4-hydroxylase subunit beta & $\mathrm{N}$ & N & & \\
\hline 143 & IGFBP3 & 7 & Insulin-like growth factor binding protein 3 & $\mathrm{~N}$ & $\mathrm{~N}$ & & \\
\hline 144 & EIF4G2 & 11 & Eukaryotic translation initiation factor 4 gamma 2 & $\mathrm{~N}$ & $\mathrm{~N}$ & & \\
\hline 145 & CD63 & 12 & CD63 molecule & $\mathrm{N}$ & N & & \\
\hline 146 & PRDX1 & 1 & Peroxiredoxin 1 & N & N & & \\
\hline 147 & CD24 & 6 & CD24 molecule & N & $\mathrm{N}$ & & First \\
\hline 148 & YBX1 & 1 & Y-box binding protein 1 & N & $\mathrm{N}$ & & \\
\hline 149 & BEX3 & $x$ & Brain expressed $X$-linked 3 & N & $\mathrm{N}$ & & \\
\hline 150 & HSPA5 & 9 & Heat shock protein family A (Hsp70) member 5 & N & N & & \\
\hline 151 & UBC & 12 & Ubiquitin C & N & $\mathrm{N}$ & & \\
\hline 152 & MBNL3 & $x$ & Muscleblind-like splicing regulator 3 & + & $\mathrm{N}$ & & \\
\hline 153 & RPS19 & 19 & Ribosomal protein S19 & N & $\mathrm{N}$ & & \\
\hline 154 & RPS14 & 5 & Ribosomal protein S14 & N & N & & \\
\hline 155 & PFN1 & 17 & Profilin 1 & N & N & & \\
\hline 156 & $\mathrm{RHOA}$ & 3 & Ras homolog family member $\mathrm{A}$ & N & $\mathrm{N}$ & & \\
\hline 157 & $\mathrm{HIST} 1 \mathrm{H} 2 \mathrm{BD}$ & 6 & Histone cluster $1 \mathrm{H} 2 \mathrm{~B}$ family member $\mathrm{d}$ & N & $\mathrm{N}$ & & \\
\hline 158 & COX8A & 11 & Cytochrome c oxidase subunit $8 \mathrm{~A}$ & $\mathrm{~N}$ & $\mathrm{~N}$ & & \\
\hline 159 & TCEAL9 & $x$ & Transcription elongation factor A like 9 & N & $\mathrm{N}$ & & \\
\hline 160 & TMSB4X & $x$ & Thymosin beta 4, X-linked & N & $\mathrm{N}$ & & \\
\hline 161 & HSPB8 & 12 & Heat shock protein family B (small) member 8 & + & $\mathrm{N}$ & & Term \\
\hline 162 & KRT7 & 12 & Keratin 7 & N & N & & First \\
\hline 163 & TINAGL1 & 1 & Tubulointerstitial nephritis antigen-like 1 & + & $\mathrm{N}$ & & \\
\hline
\end{tabular}


Table 3 Highest expressed protein-coding genes in late first trimester placenta (Continued)

\begin{tabular}{|c|c|c|c|c|c|c|c|}
\hline \# & $\begin{array}{l}\text { Gene } \\
\text { symbol }\end{array}$ & Chr & Description & $\begin{array}{l}\text { TPM highest } \\
\text { in placenta? }^{\mathrm{a}}\end{array}$ & $\begin{array}{l}\text { Placenta } \\
\text { specific? }^{\mathrm{b}}\end{array}$ & Where else expressed? & $\begin{array}{l}\text { Upregulated in } \\
\text { first vs term }\end{array}$ \\
\hline 164 & RPL27 & 17 & Ribosomal protein L27 & $\mathrm{N}$ & N & & \\
\hline 165 & HSP90AA1 & 14 & Heat shock protein 90 alpha family class A member 1 & $N$ & N & & \\
\hline 166 & HBA2 & 16 & Hemoglobin subunit alpha 2 & $\mathrm{~N}$ & & & \\
\hline 167 & RRBP1 & 20 & Ribosome binding protein 1 & $N$ & & & \\
\hline 168 & HMGA1 & 6 & High mobility group AT-hook 1 & $\mathrm{~N}$ & N & & First \\
\hline 169 & TPI1 & 12 & Triosephosphate isomerase 1 & N & N & & \\
\hline 170 & PTMA & 2 & Prothymosin, alpha & N & N & & \\
\hline 171 & RPSA & 3 & Ribosomal protein SA & $\mathrm{N}$ & N & & \\
\hline 172 & $\mathrm{CHCHD} 2$ & 7 & Coiled-coil-helix-coiled-coil-helix domain containing 2 & N & N & & \\
\hline 173 & RPL6 & 12 & Ribosomal protein L6 & N & N & & \\
\hline 174 & PSG6 & 19 & Pregnancy specific beta-1-glycoprotein 6 & ++ & Almost & $\begin{array}{l}\text { Vascular, heart, skin, } \\
\text { intestine }\end{array}$ & Term \\
\hline 175 & UBB & 17 & Ubiquitin B & $\mathrm{N}$ & N & & \\
\hline
\end{tabular}

aplacenta enrichment: $(+)=$ placenta had the highest TPM of any body site on UniGene; $(++)=$ placenta TPM value was one order of magnitude higher than the next-highest body site; $(+++)=$ placenta TPM value was two orders of magnitude higher; $\mathrm{N}=$ placenta is not highest; No value $=$ means placenta TPM =0 on UniGene. *Validation with GSE73016 found expression in term placenta [38]

bPlacenta specificity: "specific" was strictly defined as no expression (TPM $=0$ ) in other healthy body sites on UniGene. "Almost" specific was defined as TPM >0 in six healthy body sites or less (listed). "N" means gene is expressed in at least seven other tissues (not listed)

'DEGs between early first trimester ("First", 45-59 days) and term ("Term") placenta based on microarray studies in Mikheev et al. [44]

be placenta-enriched, with UniGene-documented transcripts per million (TPM) highest in placenta compared to other tissues (Table 3). Two genes, XAGE3 and $H I S T 1 H 2 B I$, were exclusively expressed in the placenta, with HIST1H2BI showing TPM $=0$ in all healthy tissue on UniGene (Table 3). Sixteen genes (CGB3, CGB5, CGB8, HIST1H1B, HIST1H1E, HIST1H2BO, HIST1H3B, HIST 1H3C, HIST1H3F, HIST1H4B, HIST1H4D, HIST1H4F, PSG3, PSG6, PSG9, and XAGE2) were almost specific to the placenta, with expression in 6 other tissues or less, most of embryonic or vascular system origin. Thirteen genes (EBI3, HIST1H1B, HIST1H1D, HIST1H1E, HIST1H2BI, HIST1H2BO, HIST1H3B, HIST1H3C, HIST1H3F, HIST 1H4B, HIST1H4C, HIST1H4D, and HIST1H4F) had no UniGene-documented placenta tissue expression, although some were expressed in many other tissues (Table 3).

To validate this potentially novel first trimester placenta expression, we cross-referenced RNA-sequencing data of term placentas (NCBI GEO Accession GSE73016) and found that EBI3 and HIST1H2BO were expressed in term placenta [38]. We also compared the top 175 proteincoding genes to a microarray study that compared early first trimester placenta (45-59 days) versus C-section delivered term placentas (NCBI GEO Accession GSE9984) and found 23 genes that are differentially expressed between early pregnancy and delivery [44]. Of the top 175 genes, 5 were significantly upregulated in first trimester (CD24, COL6A2, ENO1, HMGA1, KRT7) and 18 genes were significantly upregulated in term placenta (ADAM12,
CYP19A1, EBI3, FBLN1, GDF15, HSPB8, KISS1, PAPPA, PAPPA2, PSG1, PSG3, PSG4, PSG6, PSG9, S100A9, S100P, $S D C 1, S L C 2 A 1)$ [44]. The XAGE2, XAGE3, CGB family, and histone-encoding genes were not identified as gestationally different. Overall, there remained 11 histone-encoding genes highly expressed in late first trimester placenta that showed no UniGenedocumented placenta expression (Table 3) nor expression in term placenta [38, 45], suggesting they are unique to first trimester placenta and potentially critical for early placentation.

\section{Sex differences exist in placenta gene expression}

Among the 39 first trimester placenta evaluated, there were 17 females and 22 males. There was no statistically significant difference in maternal age, maternal race, maternal ethnicity, pre-pregnancy body mass index (BMI), gestational age at CVS, or gestational age at delivery (Table 4). Mean CRL was $58.8 \pm 12.8 \mathrm{~mm}$ and $51.8 \pm$ $10.2 \mathrm{~mm}(P=0.0714)$ for females and males, respectively. Thus, there was no difference in fetal size in the late first trimester among the sexes, based on CRL, adjusted or unadjusted. There were also no statistically significant differences in second trimester parameters. However, there was a significant difference in the birth weight among the sexes, with males being heavier at $3715 \pm 407 \mathrm{~g}$ versus $3176 \pm 405 \mathrm{~g}$ for females $(P=0.0006)$ (Table 4$)$. Even in a multivariate linear regression after adjustment for maternal age, gestational age at delivery and pre-pregnancy BMI, male 
Table 4 Subject demographics analysis

\begin{tabular}{|c|c|c|c|c|}
\hline Demographics of patient samples for RNA-Seq & $\begin{array}{l}\text { Female infants } \\
(n=17)\end{array}$ & $\begin{array}{l}\text { Male infants } \\
(n=22)\end{array}$ & $P$ value & $\frac{n}{n}$ \\
\hline Maternal age, years & $39.4 \pm 1.8$ & $39.4 \pm 2.9$ & $0.9974^{a}$ & 39 \\
\hline Maternal race: Caucasian & $17(100 \%)$ & $22(100 \%)$ & N/A & 39 \\
\hline Maternal ethnicity: Hispanic & $2(12 \%)$ & $3(14 \%)$ & $1^{b}$ & 39 \\
\hline Maternal pre-pregnancy BMI, kg/m² & $22.7 \pm 3.5$ & $21.9 \pm 2.8$ & $0.4416^{\mathrm{a}}$ & 39 \\
\hline Gestational age at CVS, days & $86 \pm 7$ & $81 \pm 6$ & $0.0503^{\mathrm{a}}$ & 39 \\
\hline Crown-rump length (CRL), mm & $58.8 \pm 12.8$ & $51.8 \pm 10.2$ & $0.0714^{\mathrm{a}}$ & 37 \\
\hline Normal karyotype at CVS & $17(100 \%)$ & $22(100 \%)$ & N/A & 39 \\
\hline Gestational age at delivery, days & $272 \pm 10$ & $276 \pm 11$ & $0.1958^{\mathrm{a}}$ & 37 \\
\hline Birth weight, grams & $3176 \pm 405$ & $3715 \pm 407$ & $0.0006^{\mathrm{a} *}$ & 34 \\
\hline
\end{tabular}

*Significant

aStudent's $t$ test

${ }^{\mathrm{b}}$ Fisher's exact test

infants were $401 \mathrm{~g}$ heavier $(P=0.003,95 \%$ CI $146-$ 657) than female infants at birth (Table 4). This sexual dimorphism in birth weight is consistent with existing literature [15].

Principal components analysis demonstrated that male and female samples separate into two clusters along a diagonal between the first and second principal components (Additional file 1A). Using DESeq2 on the unfiltered RNA-seq data, volcano and MA plots display the separation of RNA-seq results, with the largest foldchanges in expression coming from Y-linked genes as expected then $\mathrm{X}$-linked genes (Additional file 1B, C). We identified 112 genes significantly different between males and females after adjusting for multiple comparisons and filtering by Benjamini-Hochberg false discovery rate, FDR $<0.05$ (Additional file $1 \mathrm{~B}$ and Additional file 4). Cell markers for different trophoblasts cell types were not significantly different, suggesting that male and female CVS samples contained similar cell types (Additional file 5). Of 112 genes, 58 genes also meet our FPKM $>1.281$ selection criteria, leaving 35 expressed genes significantly upregulated in females and 23 expressed genes significantly upregulated in males (Fig. 2a, Tables 5 and 6).

As expected, most of the 58 differentially expressed genes (DEGs) are from the sex chromosomes (40/58; 69.0\%). Twenty-five genes (43.1\% of all DEGs) come from the $\mathrm{X}$ chromosome (18 protein-coding, 6 long non-coding, and 1 short non-coding), with 22 upregulated in females (Fig. 2b). Of all the X-linked genes upregulated in females, the majority, $13 / 22$ (59.1\%), were previously described to escape $\mathrm{X}$ chromosome inactivation $(\mathrm{XCI})$. Of the remaining 9 genes, 4 underwent $\mathrm{XCI}$ and 5 had unknown XCI status [42, 46, 47]. Several (18/ $40 ; 31.0 \%)$ of the sex chromosome genes differentially expressed among the sexes in the late first trimester were identified to be sex different in term placenta tissue
(Additional file 6), suggesting that they are important throughout gestation [20]. Differentially expressed autosome genes in the late first trimester identified here were unique and did not overlap with term placenta (Table 5 and 6, Additional file 6) nor with previous microarray studies comparing early first trimester terminations versus term placenta [20,44].

We hypothesized that homologous $\mathrm{X} / \mathrm{Y}$ gene pairs would be highly represented among differentially expressed sex-linked genes. First, we examined Xlinked genes. Currently, there are 19 X-linked genes with known $\mathrm{Y}$ homologs outside of the pseudoautosomal regions where the $\mathrm{X}$ and $\mathrm{Y}$ chromosomes may recombine, PAR1 and PAR2 [43]. Six of the 19 genes $(31.6 \%)$ are upregulated in late first trimester female placentas and escape XCI in other tissue [42]. Furthermore, the corresponding $\mathrm{Y}$ homologs of these six X-linked genes are upregulated in late first trimester male placentas (Table 7).

Next, we examined Y-linked genes. Only 16 Y-linked genes were expressed in the first trimester placenta and 15 were significantly differentially expressed (FDR $<0.05$ ). Over half $(8 / 15 ; 53.3 \%)$ of differentially expressed Y-linked genes are ancestral (DDX3Y, EIF1AY, KDM5D, RPS4Y1, TXLNGY, USP9Y, UTY, ZFY), all protein-coding except TXLNGY. These ancestral Y-linked genes are derived from the autosome-like proto-sex chromosomes of the last common therian ancestor of marsupial and placental mammals [39, 48, 49]. The X-linked homologs of 6 of these 8 genes were significantly upregulated in females, all except TXLNG $(\mathrm{FDR}=0.468)$ and USP9X $(\mathrm{FDR}=0.771)$. Males also expressed $P C D H 11 Y$, a human-specific Ylinked gene that arose from a relatively recent duplicative transposition from the $\mathrm{X}$ chromosome, 6 million years ago $[49,50]$. $P C D H 11 X$, the X-linked homolog of $P C D H 11 Y$, was not differentially expressed between males and females in the first trimester placenta 

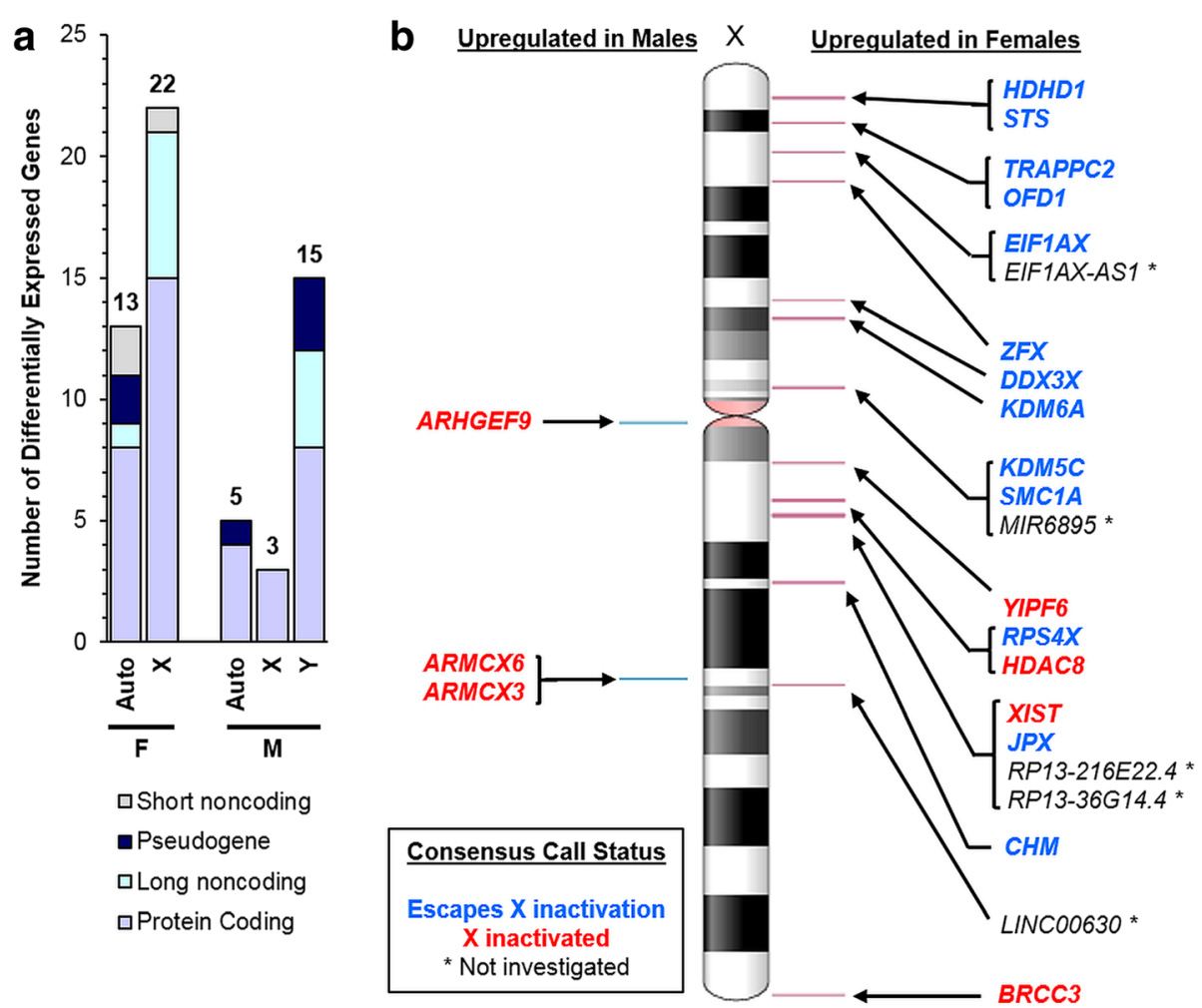

Fig. 2 Significantly differentially expressed genes in placenta. a Chromosome and biotype distribution of the 58 DEGs, 35 upregulated in females (F) and 23 upregulated in males (M). "Auto" = autosomal chromosomes. b Ideogram of chromosome X showing the 25 significantly differentially expressed X-linked genes between first trimester male and female placentas (FDR < 0.05). Left: 3 genes upregulated in males. Right: 22 genes upregulated in females. The consensus calls for $X$ inactivation status were adapted from Balaton et al. [42]

$(\mathrm{FDR}=0.969)$. The remaining Y-linked genes were noncoding (Table 6).

For each of the 58 DEGs, gene ontologies were described and categorized into regulatory function groups when known (Fig. 3). Males and females both had upregulated genes involved in chromatin modification, transcription, splicing, translation, signal transduction, metabolic regulation, cell death and autophagy regulation, and ubiquitination. Some redundant functions were due to balanced expression of homologous $\mathrm{X} / \mathrm{Y}$ genes. However, some of these categories also had an $\mathrm{X}$-linked gene upregulated in males $(A R M C X 3)$ and differentially expressed autosome genes predominantly upregulated in females. Females also had more upregulated genes associated with signal transduction, DNA replication, cell cycle regulation, and regulation of various metabolites (e.g., hormone regulation by STS, dephosphorylation of pseudouridine $5^{\prime}$-phosphate by $P U D P)$. Males had various upregulated genes from the $\mathrm{Y}$ chromosome without known function (Fig. 3b). Three sex-linked DEGs also had differentially expressed antisense genes upregulated in the same direction (Fig. 3), forming sense/antisense pairs of unknown, potentially regulatory function.

\section{Sex differences predominate over gestational age differences in CVS samples}

We next sought to identify age-specific gene expression between early and late chorionic villus sampling (CVS) collection times. We performed subanalysis with week 11 samples (10.5-11.5 weeks at CVS) and week 13 samples (12.5-13.5 weeks at CVS) and found that samples separated by sex, but not gestational age (Additional files 7, 8, and 9). Therefore, we focused our subanalysis only on sex differences within subgroups. At week 11 , there were 30 DEGs between male $(n=9)$ and female $(n=5)$ CVS samples (Additional file 10). Three new autosome genes (STAT6, SLC4A1, LAMB3) were significantly upregulated in males (FDR $<0.05)$. The remaining 17 sex-linked DEGs were consistent with the larger 39 sample analysis: 4 female-upregulated X-linked genes (XIST, KDM6A, EIF1AX, and NUDT10) and 13 male-upregulated Y-linked genes all had $\mathrm{FDR}<0.05$ in the 39 sample analysis (Additional files 4 and 10). At week 13, there were 20 DEGs between males $(n=4)$ and females $(n=4)$ (Additional file 10). Two new autosome genes were maleupregulated (SERPINB7 and PLTP). Sex-linked DEGs were consistent with 39 sample results: female-upregulated Xlinked genes (XIST and KDM5C), male-upregulated X- 
Table 5 Genes significantly upregulated in females (downregulated in males)

\begin{tabular}{|c|c|c|c|c|c|c|}
\hline Gene symbol & Chr & Description & $\begin{array}{l}\text { Fold-change } \\
\text { (F/M) }\end{array}$ & $\mathrm{FDR}^{\mathrm{a}}$ & FPKM in $\mathrm{F}$ & FPKM in $M$ \\
\hline $\mathrm{XIST}$ & $x$ & X inactive specific transcript (non-protein coding) & 26.909 & 1.83E-172 & 21.68 & 0.06 \\
\hline KDM6A & $x$ & Lysine demethylase $6 \mathrm{~A}$ & 1.674 & $1.36 \mathrm{E}-52$ & 11.71 & 6.53 \\
\hline PUDP & $x$ & Pseudouridine 5'-phosphatase & 1.653 & 1.08E-21 & 12.47 & 7.04 \\
\hline EIF1AX-AS1 & $x$ & EIF1AX antisense RNA 1 & 1.598 & 0.00204 & 2.84 & 1.34 \\
\hline MIR6895 & $x$ & MicroRNA 6895 & 1.589 & 0.0353 & 2.25 & 0.63 \\
\hline ZNF300 & 5 & Zinc finger protein 300 & 1.580 & 0.0257 & 1.44 & 0.60 \\
\hline RPL23AP11 & 14 & Ribosomal protein L23a pseudogene 11 & 1.567 & 0.0302 & 2.40 & 1.27 \\
\hline Y_RNA & 5 & Y RNA & 1.564 & 0.0438 & 7.13 & 3.63 \\
\hline MIR548B & 6 & MicroRNA 548b & 1.552 & 0.0257 & 2.00 & 0.34 \\
\hline EIF1AX & $x$ & Eukaryotic translation initiation factor $1 \mathrm{~A}, \mathrm{X}$-linked & 1.539 & 4.17E-10 & 20.05 & 11.57 \\
\hline RP13-216E22.4 & $x$ & [No description] & 1.538 & 0.000323 & 4.32 & 2.57 \\
\hline IQCJ-SCHIP1-AS1 & 3 & IQCJ-SCHIP1 read through antisense RNA 1 & 1.518 & 0.0105 & 2.94 & 1.80 \\
\hline STS & $x$ & Steroid sulfatase (microsomal), isozyme S & 1.515 & 3.89E-05 & 93.88 & 57.49 \\
\hline RPS4X & $x$ & Ribosomal protein S4, X-linked & 1.490 & 8.09E-09 & 336.10 & 209.62 \\
\hline RP13-36G14.4 & $x$ & [no description] & 1.471 & 0.0481 & 1.64 & 1.10 \\
\hline DDX3X & $x$ & DEAD-box helicase 3, X-linked & 1.434 & 3.65E-18 & 74.41 & 47.42 \\
\hline ITGB8 & 7 & Integrin subunit beta 8 & 1.402 & 0.0372 & 7.96 & 5.14 \\
\hline ZFX & $x$ & Zinc finger protein, $X$-linked & 1.392 & 1.81E-07 & 19.33 & 12.77 \\
\hline RAB30 & 11 & RAB30, member RAS oncogene family & 1.385 & 0.0494 & 2.18 & 1.37 \\
\hline$J P X$ & $x$ & JPX transcript, XIST activator (non-protein coding) & 1.379 & 4.32E-08 & 5.40 & 3.62 \\
\hline KDM5C & $x$ & Lysine demethylase $5 \mathrm{C}$ & 1.364 & 0.00728 & 24.18 & 15.82 \\
\hline SMC1A & $x$ & Structural maintenance of chromosomes $1 \mathrm{~A}$ & 1.360 & 0.000196 & 14.72 & 9.70 \\
\hline TRAPPC2 & $x$ & Trafficking protein particle complex 2 & 1.358 & 0.000204 & 4.40 & 2.97 \\
\hline TBC1D32 & 6 & TBC1 domain family member 32 & 1.349 & 0.00774 & 3.74 & 2.56 \\
\hline LINC00643 & 14 & Long intergenic non-protein coding RNA 643 & 1.337 & 0.0416 & 6.02 & 4.01 \\
\hline RASSF6 & 4 & Ras association domain family member 6 & 1.310 & 0.00516 & 12.23 & 8.74 \\
\hline LINC00630 & $x$ & Long intergenic non-protein coding RNA 630 & 1.295 & 0.0112 & 2.92 & 2.13 \\
\hline BRCC3 & $x$ & BRCA1/BRCA2-containing complex subunit 3 & 1.291 & 0.000701 & 10.84 & 7.50 \\
\hline $\mathrm{CHM}$ & $x$ & CHM, Rab escort protein 1 & 1.269 & 0.0122 & 4.49 & 3.23 \\
\hline YIPF6 & $x$ & Yip1 domain family member 6 & 1.267 & 0.0209 & 15.97 & 11.67 \\
\hline ZNF799 & 19 & Zinc finger protein 799 & 1.261 & 0.0172 & 4.33 & 3.26 \\
\hline HDAC8 & $x$ & Histone deacetylase 8 & 1.260 & 0.000121 & 3.96 & 2.92 \\
\hline PHTF1 & 1 & Putative homeodomain transcription factor 1 & 1.234 & 0.0274 & 4.78 & 3.64 \\
\hline OFD1 & $x$ & OFD1, centriole and centriolar satellite protein & 1.230 & 0.00137 & 9.05 & 6.90 \\
\hline OSBPL3 & 7 & Oxysterol binding protein-like 3 & 1.223 & 0.00921 & 4.80 & 3.73 \\
\hline
\end{tabular}

Sorted by fold-change. $F=$ females. $M=$ males

aenjamini-Hochberg false discovery rate

linked genes (ARMCX3 and ARMCX3), and 14 Y-linked genes (all except PSMA6P1).

\section{Discussion}

Normative transcriptome and highest expressed genes consistent with a time of highly regulated rapid growth The first trimester placenta is in a state of high transcriptional activity [7, 51]. We identified 14,250 genes expressed as part of the normative late first trimester placenta transcriptome. Chromosome 19 has the highest proportion of expression genes among all human chromosomes [52, 53], and this is reflected in the placental transcriptome as well. Chromosome $\mathrm{Y}$ was least expressed. However, the placenta transcriptome was enriched for ancestral Y-linked genes that have survived on the chromosome $\mathrm{Y}$ since its evolution from an 
Table 6 Genes significantly upregulated in males (downregulated in females)

\begin{tabular}{|c|c|c|c|c|c|c|}
\hline Gene symbol & Chr & Description & Fold-change (M/F) & $\mathrm{FDR}^{\mathrm{a}}$ & FPKM in $\mathrm{F}$ & FPKM in $M$ \\
\hline RPS4Y1 & Y & Ribosomal protein S4, Y-linked 1 & 203.657 & 0 & 0.01 & 61.83 \\
\hline DDX3Y & Y & DEAD-box helicase 3, Y-linked & 167.730 & 0 & 0.01 & 21.83 \\
\hline KDM5D & Y & Lysine demethylase 5D & 160.898 & 0 & 0.00 & 6.73 \\
\hline USP9Y & Y & Ubiquitin specific peptidase 9, Y-linked & 110.661 & 0 & 0.00 & 6.27 \\
\hline ZFY & Y & Zinc finger protein, Y-linked & 83.865 & 0 & 0.00 & 5.66 \\
\hline UTY & Y & Ubiquitously transcribed tetratricopeptide repeat containing, Y-linked & 83.286 & 0 & 0.00 & 7.99 \\
\hline TTY15 & Y & Testis-specific transcript, Y-linked 15 (non-protein coding) & 78.249 & 0 & 0.00 & 4.42 \\
\hline EIF1AY & Y & Eukaryotic translation initiation factor $1 \mathrm{~A}, \mathrm{Y}$-linked & 62.683 & 0 & 0.01 & 6.11 \\
\hline LINC00278 & Y & Long intergenic non-protein coding RNA 278 & 15.671 & $1.51 \mathrm{E}-127$ & 0.02 & 5.44 \\
\hline TTY14 & Y & Testis-specific transcript, Y-linked 14 (non-protein coding) & 13.548 & 4.73E-108 & 0.01 & 1.69 \\
\hline ANOS2P & Y & Anosmin 2, pseudogene & 12.295 & $2.38 \mathrm{E}-102$ & 0.01 & 1.28 \\
\hline PCDH11Y & Y & Protocadherin 11 Y-linked & 12.042 & 7.38E-132 & 0.08 & 2.30 \\
\hline TXLNGY & Y & Taxilin gamma pseudogene, Y-linked & 9.714 & $1.44 \mathrm{E}-75$ & 0.00 & 1.37 \\
\hline ZFY-AS1 & Y & ZFY antisense RNA 1 & 8.056 & $7.64 \mathrm{E}-66$ & 0.02 & 1.97 \\
\hline PSMA6P1 & Y & Proteasome subunit alpha 6 pseudogene 1 & 3.482 & $6.21 \mathrm{E}-27$ & 0.40 & 2.02 \\
\hline ARMCX6 & $x$ & Armadillo repeat containing, $\mathrm{X}$-linked 6 & 1.795 & 7.62E-06 & 1.78 & 3.95 \\
\hline MTRNR2L8 & 11 & MT-RNR2-like 8 & 1.613 & 0.0201 & 2.90 & 4.88 \\
\hline ARMCX3 & $x$ & Armadillo repeat containing, $\mathrm{X}$-linked 3 & 1.609 & 0.000255 & 6.70 & 11.93 \\
\hline HMGCS2 & 1 & 3-hydroxy-3-methylglutaryl-CoA synthase 2 & 1.603 & 0.035 & 1.99 & 4.27 \\
\hline FRG1JP & 9 & FSHD region gene 1 family member J, pseudogene & 1.507 & 0.0209 & 1.36 & 2.27 \\
\hline HSPB1 & 7 & Heat shock protein family B (small) member 1 & 1.486 & 0.0482 & 41.94 & 65.09 \\
\hline TLE2 & 19 & Transducin-like enhancer of split 2 & 1.409 & 0.0495 & 2.61 & 3.67 \\
\hline ARHGEF9 & $x$ & Cdc42 guanine nucleotide exchange factor 9 & 1.344 & 0.0416 & 1.28 & 1.75 \\
\hline
\end{tabular}

Sorted by fold-change. $F=$ females. $M=$ males

${ }^{a}$ Benjamini-Hochberg false discovery rate

autosome precursor, with $47.1 \%$ (8 of 17 total ancestral genes present in humans) expressed in late first trimester [39]. Expressed Y-linked genes were located in previously identified euchromatin regions, where DNA is less condensed and more accessible for transcription [54], consistent with the placental state of high transcriptional activity. Most $(10 / 16 ; 62.5 \%)$ of expressed Y-linked genes have current or previous homology with the $\mathrm{X}$ chromosome. All eight protein-coding genes and one pseudogene (TXLNGY) have X homologs [54]. Another pseudogene (ANOS2P) is a "degraded homolog" of X-linked ANOS1 gene, inactivated by a frameshift [55]. Notably, the testisdetermining factor (SRY gene) is not expressed in the first trimester placenta, although it is expressed in male fetal cells as early as pre-implantation [56]. Together, this suggests that $\mathrm{Y}$ chromosome expression in the first trimester placenta may be supplementing the $\mathrm{X}$ chromosome in a pseudo-autosomal fashion, necessary for placental functions that may be distinct from fetal sex development.

The biotypes of expressed genes were skewed across FPKM distribution, with the highest expressed genes predominantly protein-coding genes. Short non-coding genes were expected to be underrepresented because the RNA extraction method is optimized for transcripts 200 nucleotides or above. Nevertheless, the two most expressed genes by FPKM were both short non-coding genes, 7SK and RN7SL2. Human 7SK small nuclear RNA is abundant in animal tissue and critical for transcription elongation [57]. RN7SL2 encodes 7SL RNA, an essential component of the signal recognition particle responsible for targeting proteins to the endoplasmic reticulum, a cell organelle important for protein secretion $[58,59]$. Since placentation is a time of rapid cell proliferation and essential fetalmaternal communication, the high abundance of $7 S K$ and RN7SL2 may be important to reduce growth restriction and facilitate protein secretion.

Gene enrichment analysis and upstream analysis of the top two quartiles of expressed genes showed a consistent pattern of expression in the placenta. Among the top $25 \%$ of genes, protein synthesis and cell growth pathways and functions were significantly enriched. Mitochondrial function and metabolic pathways were also significantly enriched, including the sirtuin signaling pathway involved in nutrient sensing and cellular 
Table 7 Chromosome X-linked genes with Y homologs

\begin{tabular}{|c|c|c|c|c|c|c|c|}
\hline Chr $X$ gene & $\mathrm{XCl}$ & $\Delta \mathrm{F}$ & Chr X location & Gene descriptions & Chr Y gene & $\Delta M$ & Chr Y location \\
\hline NLGN4X & $\mathrm{E}$ & & p22.33 & Neuroligin 4 & NLGN4Y & A & $\mathrm{q} 11.221$ \\
\hline TBL1X & $E$ & & p22.3 & Tranducin $\beta$-like 1 & TBL1Y & A & p11.2 \\
\hline AMELX & - & & p22.31-p22.1 & Amelogenin & AMELY & A & p11.2 \\
\hline TMSB4X & - & & $q 21.3-q 22$ & Thymosin $\beta 4$ & TMSB4Y & A & q11.221 \\
\hline TXLNG & E & A & p22.2 & Taxilin $\gamma$ & TXLNGY & $P$ & $q 11.222-q 11.223$ \\
\hline EIF1AX & $E$ & $P, A$ & p22.12 & Eukaryotic translation initiation factor $1 \mathrm{~A}$ & EIF1AY & $P, A$ & q11.223 \\
\hline ZFX & $E$ & $P, A$ & p21.3 & Zinc finger protein & ZFY & $P, A$ & p11.2 \\
\hline USP9X & $E$ & & p11.4 & Ubiquitin specific peptidase 9 & USP9Y & $P, A$ & q11.221 \\
\hline DDX3X & E & $P, A$ & p11.3-p11.23 & DEAD-box helicase 3 & DDX3Y & $P, A$ & q11.221 \\
\hline KDM6A & E & $P, A$ & p11.2 & Lysine demethylase & UTY & $P, A$ & $q 11.221$ \\
\hline TSPYL2 & $\mathrm{S}$ & & p11.2 & Testis-specific protein Y-encoded (like 2) & TSPY1 & & $\mathrm{p} 11.2$ \\
\hline KDM5C & $E$ & $P, A$ & p11.22-p11.21 & Lysine demethylase & KDM5D & $P, A$ & q11.223 \\
\hline RPS4X & E & $P, A$ & q13.1 & Ribosomal protein $\mathrm{S} 4$ & RPS4Y1 & $P, A$ & p11.2 \\
\hline TGIF2LX & - & & q21.31 & TGFß-induced factor homeobox2-like & TGIF2LY & & p11.2 \\
\hline PCDH11X & E & & q21.3 & Protocadherin 11 & PCDH11Y & $P, A$ & p11.2 \\
\hline XKRX & S & & q22.1 & XK, Kell blood group complex subunit-related & XKRY & & q11.222 \\
\hline RBMX & S & & q26.3 & RNA binding motif & RBMY & & q11.223 \\
\hline sox3 & S & & q27.1 & SRY-box $3 /$ sex determining region $Y$ & SRY & A & p11.2 \\
\hline HSFX1 & - & & q28 & Heat shock transcriptional factor family & HSFY1 & & q11.222 \\
\hline
\end{tabular}

Table of all $\mathrm{X} / \mathrm{Y}$ homologs (excluding $\mathrm{Y}$ pseudogenes), sorted by location of $\mathrm{X}$-linked genes. $\mathrm{X}$ chromosome inactivation (XCl) status is indicated as $\mathrm{S}$ (silenced/inactivated), $\mathrm{E}$ (escapes inactivation), or "-" if unstudied, as reviewed in Balaton et al. [42]. Sexually dimorphic expression is noted when genes are upregulated in first trimester placenta (" $\mathrm{P}$ ", this study) and adult postmortem tissue ("A", Mele et al. 2015) [84]. The X genes listed are located within non-PAR regions. $\boldsymbol{\Delta}$ F = upregulated in females. $\Delta \mathrm{M}=$ upregulated in males

metabolism [60]. Second quartile genes were enriched for hormone signaling pathways, and their binding partners (e.g., estradiol hormone) were found upstream of genes from the highest expressed quartile. Pregnancy maintenance is dependent on the interplay between hormone signaling and growth signals. The maintenance of the corpus luteum and its production of hormone during the first 8 weeks depends on trophectodermoriginated human chorionic gonadotropin (hCG) hormone [61], after which the placental production of

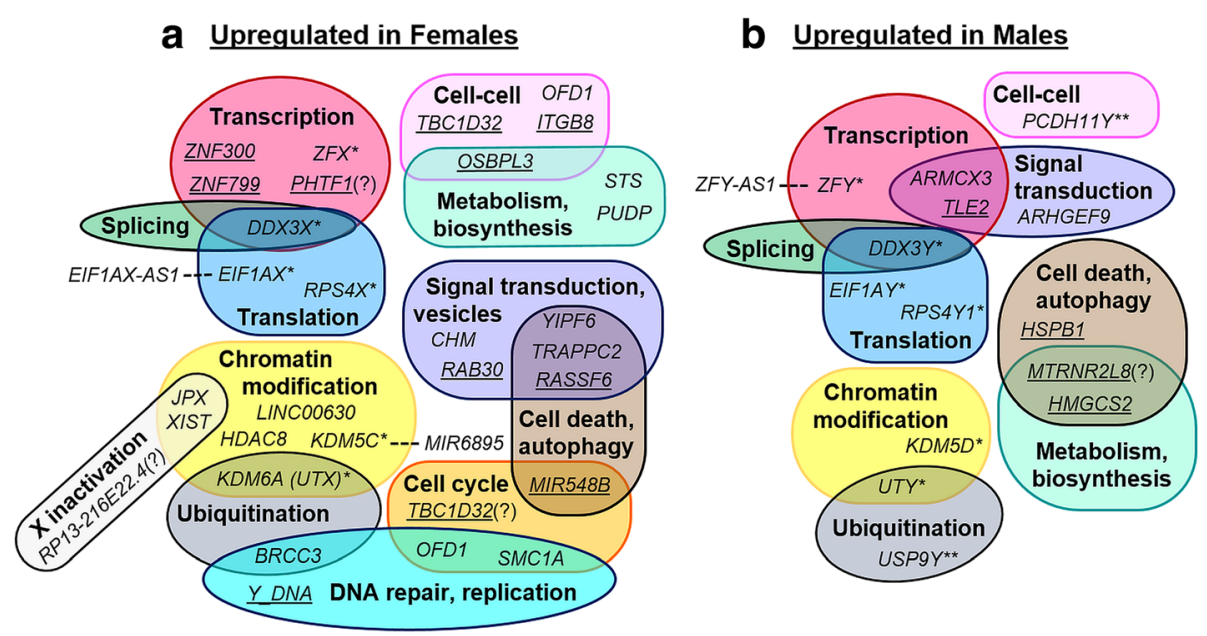

Fig. 3 Functions of significantly differentially expressed genes in placenta. Venn diagrams of function categories for DEGs with known gene ontologies $[39,84,85,93,97,98,100-106,114-126]$. Autosome genes are underlined. Sense/antisense pairs of DEGs are linked with dashed lines, with antisense genes outside the circles. *Homologous XY genes. ${ }^{* *}$ Genes with an X-linked homolog which is not differentially expressed. Functions predicted from sequence only are denoted with "(?)" next to the gene name. Genes without known or predicted function were omitted: ANOS2P, ARMCX6, FRG1JP, IQCI-SCHIP1-AS1, LINC00278, LINC00643, PSMA6P1, RP13-36G14.4, RPL23AP11, TTY14, TIY15, and TXLNGY. a Genes upregulated in females. b Genes upregulated in males 
progesterone and estrogen by the syncytiotrophoblast is sufficient to maintain pregnancy [62]. This is consistent with our findings demonstrating placental enrichment of hormone signaling canonical pathways with upstream regulation through estrogen. Other hormones such as insulin-like growth factors are also critical for regulating metabolism and fetal growth, especially in early pregnancy [63].

Finally, enriched pathways also show evidence of placental immune regulation activity. The JAK/STAT and STAT3 signaling pathway interacts with various cytokines, with mutations in pathway genes associated with both autoimmune and immunodeficient diseases [64]. Ceramide signaling in late first trimester placenta may be important for placental barrier integrity and maternal-fetal interactions, as ceramides are lipids important for skin's barrier properties, yet excess ceramides are associated with pregnancy-induced hypertension and preeclampsia [65, 66].

\section{Several highly expressed protein-coding genes are placenta-enriched}

Since placentation is a time of rapid growth, it was expected that essential and ubiquitously expressed genes such as ribosomal proteins would be among the highest expressed genes in the placenta. However, the highest expressed genes were not all housekeeping genes. Several had known and important functions in trophoblast growth and placental development. For example, KISS1 (highest expressed protein-coding gene, third highest overall) is frequently found in overgrown tissue and tumors, yet seems to be protective against metastasis [67, 68]. In placentation, KISS1 expression limits trophoblast invasion and migration by increasing cell adhesion to collagen, ensuring normal growth [69]. The second highest protein-coding gene was glycoprotein hormone alpha polypeptide (CGA), required for production of hCG, an important hormone produced by trophoblast cells days after conception and the biomarker for pregnancy tests [70-72]. To identify genes with placenta-specific or placenta-enriched expression, we searched for tissue expression profiles of the highest expressed protein-coding genes in the NCBI UniGene EST database for healthy tissue. Non-coding genes were omitted due to limited tissue expression data. Our in silico search showed that the highest expressed protein-coding genes in late first trimester placenta are not trivially expressed in all tissue types. Several genes have very limited tissue expression, placenta expression much higher than in other tissue, or both. Of the top 175 protein-coding genes in the first trimester, $X$ antigen family member 3 (XAGE3, also known as $P L A C 6)$ was the only gene known to be solely expressed in the placenta, out of 45 healthy body sites with possible transcript information for XAGE3 on UniGene. XAGE3 is part of a family of cancer/testis-associated antigens, although it is not expressed in either tumor or testis tissue, and its function is currently unknown [73]. BLAST of $X A G E 3$ showed high conservation among primates, poor conservation with non-primates (58\% identity or less), and no significant hits in non-placental mammals, reinforcing the uniqueness of XAGE3.

Eleven histone-encoding genes highly expressed in first trimester placenta were not found in UniGene's EST profile for placenta, have not been previously described in term placenta, and are also not among highly expressed genes in term placenta transcriptome studies $[38,44,45]$, showing that the first trimester placenta transcriptome is different from term placenta. Histones are DNA-binding proteins responsible for packaging DNA into nucleosomes, and differences in histones and histone modifications are associated with differences in DNA accessibility for transcription. The high expression of these histone-encoding genes in the first trimester placenta, but not later in gestation, suggests that the first trimester chromatin structure is unique and functions to maintain a high transcriptionally active state. Eight of these histone genes (HIST1H1B, HIST1H1E, HIST1H3B, HIST1H3C, HIST1H3F, HIST1H4B, HIST1H4D, and HIST1H4F) had very limited tissue expression in nonplacental tissue, primarily appearing in embryonic, vascular, skin, and connective tissue. Although these genes are not significantly sex-different in placenta, all eight are significantly upregulated in response to testosterone in mouse embryonic stem cells, suggesting an additional mechanism by which the prenatal hormone environment may affect placental and fetal gene expression [74]. A ninth gene, HIST1H2BI, had no significant UniGenedocumented expression in any normal tissue, showing EST count of $1 / 1092688$ (effectively TPM $=0$ ) in fetal brain, and no EST counts in any other normal or abnormal tissue. Its abundance in first trimester placenta (mean FPKM $=187, \mathrm{TPM}=564$ ) suggests that its tissue and developmental stage expression is heavily regulated. Together, these results suggest that first trimester placenta RNA transcription is tightly controlled and possibly contains a novel histone profile that is different from both term placenta and adult tissue.

\section{Sex differences exist in late first trimester placenta gene expression}

Out of 58 DEGs in the late first trimester placenta, over a third $(22 / 58 ; 37.9 \%)$ were $\mathrm{X}$-linked genes upregulated in female samples. $\mathrm{X}$ chromosome inactivation in females is initiated during early embryonic development, when one $\mathrm{X}$ chromosome is randomly silenced to achieve dosage compensation between XX females and XY males. About $12 \%$ of X-linked genes always escape $\mathrm{X}$ inactivation and about $8 \%$ additional $\mathrm{X}$-linked genes variably escape $\mathrm{X}$ 
inactivation in humans [75]. In our first trimester placenta results, over half $(13 / 22$; 59.1\%) of X-linked genes upregulated in females are reported to escape XCI based on previous studies [42]. This overrepresentation of genes known to escape $\mathrm{X}$ inactivation in female first trimester placenta may be the result of a necessary double dose effect, similar to a double dose effect described with $D A X$ [76]. This suggests that the increased expression from genes that escape XCI may be due to expression from both chromosomes.

$\mathrm{XCI}$ studies in early and term human placentas have been controversial. In earlier studies, $\mathrm{XCI}$ in the first trimester human placenta was reported either random in some studies or preferentially paternal in others [77-80]. In term placentas, relatively large patches of placental cells show either maternal or paternal inactivated $\mathrm{X}$ and the chorionic villous trees are clonally derived from only one or a few precursor cells [81, 82]. Our first trimester placenta samples consist of individual chorionic villous trees and thus might represent clonality. However, since each chorionic villus is composed of heterogenous cell populations (including trophoblast cells, mesenchymal cells, fetal endovascular cells) and different cell types have different gene expression patterns as already shown in term placenta $[20,83]$, the clonality of allelic specific expression in individual villi of the first trimester placenta remains to be determined.

Furthermore, the sexual dimorphic expression patterns of X-linked genes in the first trimester placentas from this study resemble what was identified by a recent transcriptome analysis in adult human tissues [84]. Sexually dimorphic expression patterns that are highly conserved from early placenta into adulthood may indicate important systemic sex differences. Among the 22 genes upregulated in females in first trimester placenta, 14 (63.6\%) were also upregulated in females across multiple nonplacental adult human tissues [84]. Almost all (11/14; 78.6\%) were identified to escape XCI [42], with the exception of XIST which is X-inactivated, and EIF1AX-AS1 and RP13-216E22.4 whose XCI statuses are unknown. This suggests that the upregulation of these X-linked genes in females may be due to expression from both chromosomes, beginning in the first trimester and continuing into adulthood. These genes may be critical markers of sex differences in fetal growth and development that transcends into adulthood determining sex differences in adult diseases, known as fetal origins of adult diseases.

However, not all sexually dimorphic genes matched sex differences in adult tissues [84]. There were eight Xlinked genes that were upregulated in females only in the late first trimester placentas: $B R C C 3, C H M, H D A C 8$, LINC00630, MIR6895, OFD1, RP13-36G14.4, and YIPF6. These genes play important roles in transcriptional regulation, DNA damage response, and vesicle-mediated transport, suggesting biological processes that may be sex-biased and critical for early placenta development. LINC00630 promotes cell proliferation by promoting the protein stability of histone deacetylate HDAC1 [85]. Noncoding gene MIR6895 is antisense to histone demethylaseencoding $K D M 5 C$, and thus may also regulate placental chromatin status. Among males, 13 Y-linked genes were consistent with that in the adult tissues, but pseudogenes ANOS2P and PSMA6P1 exhibited male-upregulation only in the first trimester placenta. Similar to other pseudogenes, they may act as miRNA decoys which may be important in early development [86, 87]. Three X-linked genes ARMCX3 (armadillo repeat containing, X-linked 3), ARMCX6 (armadillo repeat containing, X-linked 6), and ARHGEF9 (Cdc42 guanine nucleotide exchange factor 9) were also upregulated in male placenta, but not adult male tissues [84]. The three upregulated X-linked genes in males typically undergo $\mathrm{X}$ inactivation in other tissue, indicating that one active allele is likely sufficient for their function [42]. It remains to be determined whether the increased gene levels of these three $\mathrm{X}$-linked genes in males is due to upstream Y-linked genes or if the second $\mathrm{X}$ chromosome downregulates these genes in females. Though there is limited research, male-upregulated expression of X-linked genes has been reported previously [84, 88]. Alex3 (encoded by ARMCX3) regulates migration and invasion in tumor cells, functions which are also critical for placentation and subsequent fetal outcomes, including fetal growth [89].

Autosome genes comprised almost a third of DEGs $(18 / 58 ; 31.0 \%)$ in late first trimester placenta. In silico upstream analysis of the autosome DEGs did not yield any regulators on the sex chromosomes nor hormonal regulators that may explain the differences in expression between males and females, though this may be due to limitations of currently available data. Although many of the sex chromosome DEGs have been found in previous studies of sex differences, none of the autosome DEGs are previously identified as sex different in term placenta [20]. One autosome gene (RASSF6 on chromosome 4) was significantly upregulated in females in both late first trimester placenta and adult breast tissue [84]. Since both placenta and adult breast tissue are responsive to sex hormones, this overlap in RASSF6 makes biological sense. Overall, sex differences in autosome gene expression exist, but appear to be more subject to age- and tissue-specific variability than sex chromosome DEGs.

\section{Distribution of sexual dimorphisms along parallel pathways (X/Y homologs)}

Over a fourth $(6 / 22 ; 27.3 \%)$ of $\mathrm{X}$-linked genes upregulated in females have functional homologs on the $\mathrm{Y}$ chromosome, including EIF1AX, DDX3X, KDM5C, 
KDM6A, RPS4X, and ZFX. However, not all X-linked genes with $\mathrm{Y}$ homologs are sexually dimorphic. In fact, when examining significantly different expressed Ylinked genes in male, three Y-linked genes (PCDH11Y, TXLNGY, and USP9Y) had functional X-homologs that displayed balanced expression between the sexes. USP9Y was once thought to be essential for spermatogenesis, but men with complete deletions of $U S P 9 Y$ can be fertile [90, 91]. Polymorphisms in the $U S P 9 Y$ gene affect serum lipid profiles and coronary heart disease risk, showing that this Y-linked gene has a non-gametogenesis function [92]. The expression of three Y-linked genes in male placenta without balancing upregulation of X-linked homologs in female placentas suggests that these genes may have additional important functions for placental development that may have long-term health implications for sexually dimorphic diseases.

With pathways analysis of the 58 DEGs, we found few predicted whole pathway differences between males and females (not shown). This may be due to autosome-like expression of $\mathrm{X} / \mathrm{Y}$ gene pairs such as EIF1AX/EIF1AY and $R P S 4 X / R P S 4 Y 1$. Although the individual proteincoding genes show sexually dimorphic expression, the canonical pathways are not differentially "activated" or "inactivated," but instead function in parallel in the same direction. We saw this dimorphic-but-parallel expression in chromatin modification, transcription, splicing, and translation pathways.

\section{Functional significance of sexually dimorphic genes}

Sexually dimorphic genes are likely functionally relevant for placenta biology. Females had a greater number of upregulated genes encoding DNA binding proteins, including genes associated with chromatin modification (including $\mathrm{X}$ inactivation) and genes encoding components of centrioles and cohesin complexes (OFD1 and $S M C 1 A$, respectively) important for segregation of replicated DNA during cell division [93, 94]. Metabolismassociated upregulated genes in males were primarily involved in response to nutrient deficit. MTRNR2L8 (1.61fold upregulated in males) encodes Humanin-like 8, a small peptide homologous and sometimes identical (due to a polymorphic site) to the mitochondrially encoded Humanin peptide which promotes cell survival in ATPdeficient environments [95, 96]. Humanin promotes insulin sensitivity which may contribute to increased fetal growth seen in males [97]. HMGCS2 (1.60-fold upregulated in males) encodes an enzyme that promotes autophagy by catalyzing the first step in ketogenesis, a pathway that derives energy from lipids when carbohydrates are depleted [98]. This is consistent with previous pregnancy studies that find greater risk for nutrient deficit in males [99]. In contrast, metabolism-associated genes upregulated in females are involved in post-transcriptional modification of RNA (PUDP) and hormone biosynthesis (STS) [100, 101]. Cell adhesion, ciliogenesis, and cell-cell communication genes (OFD1, OSBL3, PCDH11Y, $T B C 1 D 32)$ were also differentially expressed, suggesting sex differences in how placenta cells interact with their environment [102-105]. ITGB8 (encodes integrin- $\beta 8$ ) promotes tumor angiogenesis and invasiveness in glioblastoma [105], functions necessary for normal first trimester development when placental cells invade maternal tissue and access maternal blood.

Differentially expressed transcription factors in first trimester may explain sex differences in later pregnancy complications. For example, ZNF300 (1.58-fold upregulated in females) encodes a transcription factor protein that shares DNA binding sites with Early growth response 1 (Egr1), a zinc finger transcription factor that is elevated in placentas of pregnancies that later develop preeclampsia, which is more common in pregnancies with a male fetus $[15,106,107]$. Increased expression of ZNF300 in females may function as a competitor of $E G R 1$ binding sites, leading to reduced rates of preeclampsia. Further studies of ZNF300 and other sex different genes are needed to understand their biological roles in placental development.

\section{Y-linked and chromatin modification genes are consistently sex different}

Gestational age-specific sex differences exist, even between 11 and 13 weeks gestation. We found that a subset of Y-linked genes are consistently expressed in late first trimester placenta regardless of gestational age and into adulthood, whereas X-linked genes and autosome genes were more variable. However, $X$-linked genes that affect chromatin modification (XIST, KDM6A, KDM5C) are conserved throughout gestation and into adulthood [84].

\section{Sex differences of fetal weight at birth}

In our cohort, male infants had higher birth weights compared to female infants, consistent with known sex differences at birth $[108,109]$. Sex differences in embryonic and fetal growth rates have been long recognized [110], but the underlying mechanisms remain poorly understood. Recent studies show associations between placental biomarkers and fetal growth [111-113]. Since sex differences in gene expression may be implicated in placental function, altering growth and development which can translate into sexual dimorphism of disease in adulthood, further studies are necessary to better understand the functional roles of these genes in early pregnancy.

\section{Limitations}

All subjects in this study were Caucasian. Caucasians make up the largest demographic for chorionic villus sampling tissue that is available through our prenatal 
biorepository. It remains to be seen if sex differences in placenta transcripts vary among different races. Additionally, the normative placenta transcriptome described for the late first trimester here may be underrepresenting short RNAs. We found that $6.7 \%$ of the overall transcribed genes were short non-coding RNAs. The RNA extraction method used prior to RNA-seq is optimized for RNA of lengths $>200 \mathrm{nt}$, which loses $5 \mathrm{~S}$ rRNA, tRNAs, mature miRNAs, and several other (though not all) short RNAs. Furthermore, although we compared differential expression from the late first trimester to previously published data looking at term placenta, results need to be interpreted with caution since term placenta studies were performed using microarrays with smaller sample sizes and not RNA-seq.

\section{Conclusions}

This is the largest study to date that identifies the late first trimester human placenta transcriptome, highlighting similarities and differences among the sexes. This is the earliest time point that ongoing human pregnancies that result in a live birth can be studied. We identified a group of 11 histone-encoding genes which were highly expressed in first trimester placenta, but have not been previously reported in term placenta, suggesting they may have temporally specific functions important for early pregnancy regardless of sex. When examining our cohort of patients for sex differences, we found 58 significantly differentially expressed genes with an overrepresentation of genes known to escape $\mathrm{X}$ inactivation. Many of the sexually dimorphic genes fell into a group of $\mathrm{X} / \mathrm{Y}$ gene pairs, suggesting that dosage compensation plays a role in sex differences. Gene ontology analysis of differentially expressed genes suggested molecular signaling differences that may affect pregnancy outcomes, but further study of these genes in placenta models is needed. This is the first step into the characterization of normal placental function that can lead to the development of diagnostic tests for the normal and dysfunctional placenta, as well as identification of sex differences that start in utero and may translate into sex differences of adult diseases. This study can be used to develop a sex-specific placenta atlas which will be unique to humans at this early time point in placental development.

\section{Additional files}

Additional file 1: RNA-sequencing figures for 39 CVS analysis. a Principal components analysis plot of the 39 CVS samples shows male and female clusters. Blue: male samples, red: female samples. b Volcano plot of RNA-seq results before FPKM cutoff. c MA plot of RNA-seq results before FPKM cutoff. (DOC $1937 \mathrm{~kb})$

Additional file 2: Table S1. Expressed genes in late first trimester human placenta, sorted by FPKM. Ensembl IDs, gene symbols, biotypes, FPKM values, baseMean values, gene descriptions, and statistics from DESeq2 are given. (XLS $25631 \mathrm{~kb}$ )
Additional file 3: Table S2. Top 40 upstream regulators for the top $25 \%$ of expressed genes. (DOC $84 \mathrm{~kb}$ )

Additional file 4: Table S3. Sex different genes in late first trimester placenta. DESeq2 results for FDR $<0.05$ genes before FPKM cutoff. (XLS $243 \mathrm{~kb}$ )

Additional file 5: Table S4. Cell marker expression in 39 CVS samples. Cell types in CVS tissue appear to be similar in male and female samples. No cell marker is significantly sex different. (DOC $48 \mathrm{~kb}$ )

Additional file 6: Table S5. Sex differences in CVS compared to previous term placenta study. Overlap of DEGs in the current study and DEGs in epithelium and endothelium in term placenta. (DOC 60 kb)

Additional file 7: Principal components analysis for week 11 vs week 13 subgroups. Early and late CVS collection time does not separate the samples. Green: week 11 samples. Purple: week 13 samples. (TIFF 610 kb)

Additional file 8: Week 11 DESeq2 subanalysis for sex differences. a Principal components analysis for week 11 subgroup shows male and female clusters. b Volcano plot before FPKM cutoff. c MA plot before FPKM cutoff. (DOC $1071 \mathrm{~kb}$ )

Additional file 9: Week 13 DESeq2 subanalysis for sex differences. a Principal components analysis for week 13 subgroup shows male and female clusters. b Volcano plot before FPKM cutoff. c MA plot before FPKM cutoff. (DOC $863 \mathrm{~kb}$ )

Additional file 10: Table S6 and Table S7. Sexually dimorphic genes in human placenta at weeks 11 and 13. Spreadsheet of significantly different genes for gestational age subanalysis (female vs male, FDR < 0.05). Table S6: week 11. Table S7: week 13. (XLS $70 \mathrm{~kb}$ )

\section{Abbreviations}

BMI: Body mass index; CRL: Crown-rump length; CVS: Chorionic villus sampling; DEG: Differentially expressed gene; EST: Expressed sequence tag; FDR: False discovery rate; FPKM: Fragments per kilobase per million mapped fragments; GEO: Gene Expression Omnibus; hCG: Human chorionic gonadotropin; IPA: Ingenuity Pathways Analysis; IUGR: Intrauterine growth restrictions; NCBI: National Center for Biotechnology Information; RIN: RNA integrity number; TPM: Transcripts per million; XCI: X chromosome inactivation

\section{Acknowledgements}

The authors would like to thank Kerlly Castellano, Danielle Tripoli, and Lindsay L. Kroener, MD for help with patient recruitment and sample selection.

\section{Funding}

We gratefully acknowledge the support from the National Institutes of Health, specifically the Eunice Kennedy Shriver National Institute of Child Health and Human Development R01 grants to MDP (R01HD074368, R01HD091773) and the Ruth L. Kirschstein National Research Service Award to TLG (T32DK007770). The content is solely the responsibility of the authors and does not necessarily represent the official views of the National Institutes of Health. The funding agency was not involved in the design, analysis, or interpretation of the data reported.

\section{Availability of data and materials}

The RNA-sequencing data has been deposited in NCBI GEO Accession GSE109082.

\section{Authors' contributions}

MDP conceived and designed the study and critically reviewed the manuscript. TLG processed the villi samples, performed sample quality analysis, gene ontology and pathways analysis, gene distributions and clusters analysis, UniGene analysis, and wrote the manuscript. TS performed X inactivation analysis and contributed to the written manuscript. BL processed the villi samples for RNA-seq analysis. AFK, CRF, SSR, and SDT performed RNA-seq alignments, statistics, and sequencing quality analysis. LS and ETW analyzed patient demographics. YIC, JR, and MG critically reviewed and contributed to the final manuscript. RB contributed to sample collection. JW performed all CVS sampling and provided all tissue samples for the study. All authors read and approved the final manuscript. 


\section{Ethics approval and consent to participate}

Patients were recruited with informed written consent. All protocols were performed in accordance with the institutional review board's guidelines at the Cedars-Sinai Medical Center under IRB Protocols Pro00006806 (prenatal repository) and Pro00008600 (differential gene expression of early placenta).

\section{Consent for publication}

Not applicable.

\section{Competing interests}

The authors declare that they have no competing interests.

\section{Publisher's Note}

Springer Nature remains neutral with regard to jurisdictional claims in published maps and institutional affiliations.

\section{Author details}

'Department of Obstetrics and Gynecology, Division of Reproductive Endocrinology and Infertility, Cedars-Sinai Medical Center, Los Angeles, CA, USA. ${ }^{2}$ Center for Public Health Genomics, University of Virginia, Charlottesville, VA, USA. ${ }^{3}$ Division of Reproductive Endocrinology and Infertility, UCLA David Geffen School of Medicine, Los Angeles, CA, USA. ${ }^{4}$ Department of Obstetrics and Gynecology, Division of Maternal-Fetal Medicine, Cedars-Sinai Medical Center, Los Angeles, CA, USA. ${ }^{5}$ LABiomed/ Harbor-UCLA Medical Center, Torrance, CA, USA. ${ }^{6}$ Department of Medicine, Division of Endocrinology, Diabetes and Metabolism, Cedars-Sinai Medical Center, Los Angeles, CA, USA.

\section{Received: 20 October 2017 Accepted: 3 January 2018}

\section{Published online: 15 January 2018}

\section{References}

1. Redman CW, Sargent IL. Latest advances in understanding preeclampsia. Science. 2005;308(5728):1592-4

2. Pijnenborg $R$, Vercruysse $L$, Hanssens $M$. The uterine spiral arteries in human pregnancy: facts and controversies. Placenta. 2006;27(9-10):939-58.

3. Wang ET, Kathiresan ASQ, Bresee C, Greene N, Alexander C, Pisarska MD. Abnormal implantation after fresh and frozen in vitro fertilization cycles. Fertil Steril. 2017;107(5):1153-8.

4. Sundheimer LW, Pisarska MD. Abnormal placentation associated with infertility as a marker of overall health. Semin Reprod Med. 2017;35(3):205-16.

5. Kroener L, Wang ET, Pisarska MD. Predisposing factors to abnormal first trimester placentation and the impact on fetal outcomes. Semin Reprod Med. 2016:34(1):27-35

6. Ball MP, Li JB, Gao Y, Lee JH, LeProust EM, Park IH, et al. Targeted and genome-scale strategies reveal gene-body methylation signatures in human cells. Nat Biotechnol. 2009;27(4):361-8.

7. Chu T, Handley D, Bunce K, Surti U, Hogge WA, Peters DG. Structural and regulatory characterization of the placental epigenome at its maternal interface. PLoS One. 2011;6(2):e14723.

8. Schroeder DI, Blair JD, Lott P, Yu HO, Hong D, Crary F, et al. The human placenta methylome. Proc Natl Acad Sci U S A. 2013;110(15):6037-42.

9. Bianco-Miotto T, Mayne BT, Buckberry S, Breen J, Rodriguez Lopez CM, Roberts $\mathrm{CT}$. Recent progress towards understanding the role of DNA methylation in human placental development. Reproduction. 2016;152(1):R23-30.

10. Roberts CT. IFPA award in placentology lecture: complicated interactions between genes and the environment in placentation, pregnancy outcome and long term health. Placenta. 2010;31(Suppl):S47-53.

11. Segars JH, Niblack GD, Osteen KG, Rogers BJ, Wentz AC. The human blastocyst produces a soluble factor(s) that interferes with lymphocyte proliferation. Fertil Steril. 1989;52(3):381-7.

12. O'Tierney-Ginn PF, Lash GE. Beyond pregnancy: modulation of trophoblast invasion and its consequences for fetal growth and long-term children's health. J Reprod Immunol. 2014;104-105:37-42.

13. Whitley GSJ, Cartwright JE. Trophoblast-mediated spiral artery remodelling: a role for apoptosis. J Anat. 2009:215(1):21-6.

14. Di Renzo GC, Rosati A, Sarti RD, Cruciani L, Cutuli AM. Does fetal sex affect pregnancy outcome? Gender Medicine. 2007:4(1):19-30.

15. Verburg PE, Tucker G, Scheil W, Erwich JJHM, Dekker GA, Roberts CT, et al. Sexual dimorphism in adverse pregnancy outcomes - a retrospective Australian population study 1981-2011. PLoS One. 2016;11(7):e0158807.
16. Bukowski R, Smith GCS, Malone FD, Ball RH, Nyberg DA, Comstock CH, et al. Human sexual size dimorphism in early pregnancy. Am J Epidemiol. 2007; 165(10):1216-8

17. Peacock JL, Marston L, Marlow N, Calvert SA, Greenough A. Neonatal and infant outcome in boys and girls born very prematurely. Pediatr Res. 2012 71(3):305-10.

18. Byrne J, Warburton D, Opitz JM, Reynolds JF. Male excess among anatomically normal fetuses in spontaneous abortions. Am J Med Genet. 1987;26(3):605-11.

19. Sood R, Zehnder JL, Druzin ML, Brown PO. Gene expression patterns in human placenta. Proc Natl Acad Sci. 2006;103(14):5478-83.

20. Cvitic S, Longtine MS, Hackl H, Wagner K, Nelson MD, Desoye G, et al. The human placental sexome differs between trophoblast epithelium and villous vessel endothelium. PLoS One. 2013;8(10):e79233.

21. Buckberry S, Bianco-Miotto T, Bent SJ, Dekker GA, Roberts CT. Integrative transcriptome meta-analysis reveals widespread sex-biased gene expression at the human fetal-maternal interface. Mol Hum Reprod. 2014;20(8):810-9.

22. Singh K, Williams J, Brown J, Wang ET, Lee B, Gonzalez TL, et al. Upregulation of microRNA-202-3p in first trimester placenta of pregnancies destined to develop severe preeclampsia, a pilot study. Pregnancy Hypertens. 2017;10:7-9.

23. Barker DJ, Eriksson JG, Forsen T, Osmond C. Fetal origins of adult disease: strength of effects and biological basis. Int J Epidemiol. 2002;31(6):1235-9.

24. Alati R, Lawlor DA, Mamun AA, Williams GM, Najman JM, O'Callaghan M, et al. Is there a fetal origin of depression? Evidence from the mater university study of pregnancy and its outcomes. Am J Epidemiol. 2007; 165(5):575-82

25. Carter AM. Animal models of human placentation-a review. Placenta. 2007;28:S41-S7

26. Hou S, Chen J-F, Song M, Zhu Y, Jan YJ, Chen SH, et al. Imprinted NanoVelcro microchips for isolation and characterization of circulating fetal trophoblasts: toward noninvasive prenatal diagnostics. ACS Nano. 2017;11(8):8167-77.

27. Pisarska MD, Akhlaghpour M, Lee B, Barlow GM, Xu N, Wang ET, et al. Optimization of techniques for multiple platform testing in small, precious samples such as human chorionic Villus sampling. Prenat Diagn. 2016:36:1061-70.

28. Dobin A, Davis CA, Schlesinger F, Drenkow J, Zaleski C, Jha S, et al. STAR: ultrafast universal RNA-seq aligner. Bioinformatics. 2013;29(1):15-21.

29. Liao Y, Smyth GK, Shi W. featureCounts: an efficient general purpose program for assigning sequence reads to genomic features. Bioinformatics. 2014;30(7):923-30

30. Anders S, Huber W. Differential expression analysis for sequence count data. Genome Biol. 2010:11(10):R106-R.

31. Love Ml, Huber W, Anders S. Moderated estimation of fold change and dispersion for RNA-seq data with DESeq2. Genome Biol. 2014;15(12):550.

32. Team RC. R: a language and environment for statistical computing. Vienna: R Foundation for Statistical Computing: 2015.

33. NCBI Resource Coordinators. Database Resources of the National Center for Biotechnology Information. Nucleic Acids Res. 2017:45(D1):D12-d7.

34. Xu N, Barlow GM, Cui J, Wang ET, Lee B, Akhlaghpour M, et al. Comparison of genome-wide and gene-specific DNA methylation profiling in firsttrimester chorionic villi from pregnancies conceived with infertility treatments. Reprod Sci. 2017:24(7):996-1004.

35. Lee B, Kroener LL, Xu N, Wang ET, Banks A, Williams J III, et al. Function and hormonal regulation of GATA3 in human first trimester Placentation. Biol Reprod. 2016;95(5):113

36. Mudunuri U, Che A, Yi M, Stephens RM. bioDBnet: the biological database network. Bioinformatics. 2009;25(4):555-6.

37. Kozomara A, Griffiths-Jones S. miRBase: annotating high confidence microRNAs using deep sequencing data. Nucleic Acids Res. 2014; 42(D1):D68-73.

38. Yabe S, Alexenko AP, Amita M, Yang Y, Schust DJ, Sadovsky Y. Comparison of syncytiotrophoblast generated from human embryonic stem cells and from term placentas. Proc Natl Acad Sci U S A. 2016:113(19):E2598-607.

39. Bellott DW, Hughes JF, Skaletsky H, Brown LG, Pyntikova T, Cho T-J, et al. Mammalian Y chromosomes retain widely expressed dosage-sensitive regulators. Nature. 2014;508(7497):494-9.

40. The UniProt Consortium. UniProt: the universal protein knowledgebase. Nucleic Acids Res. 2017;45(D1):D158-D69.

41. Yates A, Akanni W, Amode MR, Barrell D, Billis K, Carvalho-Silva D, et al. Ensembl 2016. Nucleic Acids Res. 2016;44(D1):D710-D6. 
42. Balaton BP, Cotton AM, Brown CJ. Derivation of consensus inactivation status for X-linked genes from genome-wide studies. Biol Sex Differ. 2015;6:35.

43. Wilson Sayres MA, Makova KD. Gene survival and death on the human Y chromosome. Mol Biol Evol. 2013;30(4):781-7.

44. Mikheev AM, Nabekura T, Kaddoumi A, Bammler TK, Govindarajan R, Hebert $M F$, et al. Profiling gene expression in human placentae of different gestational ages: an OPRU network and UW SCOR study. Reprod Sci. 2008;15(9):866-77.

45. Saben J, Zhong Y, McKelvey S, Dajani NK, Andres A, Badger TM, et al. A comprehensive analysis of the human placenta transcriptome. Placenta. 2014;35(2):125-31.

46. Cotton AM, Price EM, Jones MJ, Balaton BP, Kobor MS, Brown CJ. Landscape of DNA methylation on the $X$ chromosome reflects $C p G$ density, functional chromatin state and X-chromosome inactivation. Hum Mol Genet. 2015; 24(6):1528-39.

47. Carrel L, Willard HF. X-inactivation profile reveals extensive variability in Xlinked gene expression in females. Nature. 2005;434(7031):400-4.

48. Necsulea A, Kaessmann H. Evolutionary dynamics of coding and noncoding transcriptomes. Nat Rev Genet. 2014;15(11):734-48.

49. Cortez D, Marin R, Toledo-Flores D, Froidevaux L, Liechti A, Waters PD, et al. Origins and functional evolution of $Y$ chromosomes across mammals. Nature. 2014:508(7497):488-93.

50. Williams NA, Close JP, Giouzeli M, Crow TJ. Accelerated evolution of Protocadherin $11 \mathrm{X} / \mathrm{Y}$ : a candidate gene-pair for cerebral asymmetry and language. Am J Med Genet B Neuropsychiatr Genet. 2006;141B(6):623-33.

51. Founds SA, Conley YP, Lyons-Weiler JF, Jeyabalan A, Hogge WA, Conrad KP. Altered global gene expression in first trimester placentas of women destined to develop preeclampsia. Placenta. 2009;30(1):15-24

52. Grimwood J, Gordon LA, Olsen A, Terry A, Schmutz J, Lamerdin J, et al. The DNA sequence and biology of human chromosome 19. Nature. 2004; 428(6982):529-35.

53. McGuire AB, Rafi SK, Manzardo AM, Butler MG. Morphometric analysis of recognized genes for autism Spectrum disorders and obesity in relationship to the distribution of protein-coding genes on human chromosomes. Int J Mol Sci. 2016;17:5.

54. Lahn BT, Pearson NM, Jegalian K. The human $Y$ chromosome, in the light of evolution. Nat Rev Genet. 2001;2(3):207-16.

55. de Castro F, Seal R, Maggi R. ANOS1: a unified nomenclature for Kallmann syndrome 1 gene (KAL1) and anosmin-1. Brief Funct Genomics. 2016; elw037(2041-2657 (Electronic))

56. Fiddler M, Abdel-Rahman B, Rappolee DA, Pergament E. Expression of SRY transcripts in preimplantation human embryos. Am J Med Genet. 1995;55(1):80-4

57. Peterlin BM, Brogie JE, Price DH. 7SK snRNA: a noncoding RNA that plays a major role in regulating eukaryotic transcription. Wiley Interdiscip Rev RNA. 2012;3(1):92-103.

58. Ullu E, Weiner AM. Human genes and pseudogenes for the 7SL RNA component of signal recognition particle. EMBO J. 1984;3(13):3303-10.

59. Ellgaard L, Molinari M, Helenius A. Setting the standards: quality control in the secretory pathway. Science. 1999;286(5446):1882.

60. Covington JD, Bajpeyi S. The sirtuins: markers of metabolic health. Mol Nutr Food Res. 2016;60(1):79-91.

61. Jameson $\mathrm{J}$, Hollenberg AN. Regulation of chorionic gonadotropin gene expression. Endocr Rev. 1993;14(2):203-21.

62. Malassine A, Frendo JL, Evain-Brion D. A comparison of placental development and endocrine functions between the human and mouse model. Hum Reprod Update. 2003;9(6):531-9.

63. Pasqualini JR, Chetrite GS. The formation and transformation of hormones in maternal, placental and fetal compartments: biological implications. Horm Mol Biol Clin Invest. 2016;27(1):11-28.

64. O'Shea JJ, Holland SM, Staudt LM. JAKs and STATs in immunity, immunodeficiency, and cancer. N Engl J Med. 2013:368(2):161-70.

65. Meckfessel MH, Brandt S. The structure, function, and importance of ceramides in skin and their use as therapeutic agents in skin-care products. J Am Acad Dermatol. 2014;71(1):177-84.

66. Melland-Smith M, Ermini L, Chauvin S, Craig-Barnes H, Tagliaferro A, Todros $T$, et al. Disruption of sphingolipid metabolism augments ceramide-induced autophagy in preeclampsia. Autophagy. 2015;11(4):653-69.

67. Timologou A, Zafrakas M, Grimbizis G, Miliaras D, Kotronis K, Stamatopoulos $P$, et al. Immunohistochemical expression pattern of metastasis suppressors KAI1 and KISS1 in endometriosis and normal endometrium. Eur J Obstet Gynecol Reprod Biol. 2016;199:110-5.
68. Lee JH, Miele ME, Hicks DJ, Phillips KK, Trent JM, Weissman BE, et al. KiSS-1, a novel human malignant melanoma metastasis-suppressor gene. J Natl Cancer Inst. 1996;88(23):1731-7.

69. Babwah AV. Uterine and placental KISS1 regulate pregnancy: what we know and the challenges that lie ahead. Reproduction. 2015;150(4):R121-8.

70. Bergh PA, Navot D. The impact of embryonic development and endometrial maturity on the timing of implantation. Fertil Steril. 1992;58(3):537-42.

71. Wide L, Gemzell CA. An immunological pregnancy test. Acta Endocrinol. 1960;XXXV(II):261-7.

72. Sunder S, Lenton EA. Endocrinology of the peri-implantation period. Best Pract Res Clin Obstet Gynaecol. 2000;14(5):789-800.

73. Zendman AJW, van Kraats AA, Weidle UH, Ruiter DJ, van Muijen GNP. The XAGE family of cancer/testis-associated genes: alignment and expression profile in normal tissues, melanoma lesions and Ewing's sarcoma. Int J Cancer. 2002;99(3):361-9.

74. Bramble MS, Roach L, Lipson A, Vashist N, Eskin A, Ngun T, et al. Sexspecific effects of testosterone on the sexually dimorphic transcriptome and epigenome of embryonic neural stem/progenitor cells. Sci Rep. 2016:6:36916.

75. Balaton BP, Brown CJ. Escape artists of the X chromosome. Trends Genet. 2016;32(6):348-59.

76. Zanaria E, Bardoni B, Dabovic B, Calvari V, Fraccaro M, Zuffardi O, et al. Xp duplications and sex reversal. Philos Trans R Soc Lond Ser B Biol Sci. 1995: 350(1333):291-6.

77. Migeon BR, Do TT. In search of non-random X inactivation: studies of fetal membranes heterozygous for glucose-6-phosphate dehydrogenase. Am J Hum Genet. 1979;31(5):581-5.

78. Goto T, Wright E, Monk M. Paternal X-chromosome inactivation in human trophoblastic cells. Mol Hum Reprod. 1997;3(1):77-80.

79. Zeng SM, Yankowitz J. X-inactivation patterns in human embryonic and extra-embryonic tissues. Placenta. 2003;24(2-3):270-5.

80. Uehara S, Tamura M, Nata M, Ji G, Yaegashi N, Okamura K, et al. Xchromosome inactivation in the human trophoblast of early pregnancy. J Hum Genet. 2000:45(3):119-26.

81. Peñaherrera MS, Jiang R, Avila L, Yuen RKC, Brown CJ, Robinson WP. Patterns of placental development evaluated by $X$ chromosome inactivation profiling provide a basis to evaluate the origin of epigenetic variation. Hum Reprod. 2012:27(6):1745-53.

82. Moreira de Mello JC, de ÉSS A, Stabellini R, Fraga AM, de JES S, Sumita DR, et al. Random X inactivation and extensive mosaicism in human placenta revealed by analysis of allele-specific gene expression along the $X$ chromosome. PLoS One. 2010;5(6):e10947.

83. Tsang JCH, JSL V, Ji L, LCY P, Jiang P, Lui KO, et al. Integrative single-cell and cell-free plasma RNA transcriptomics elucidates placental cellular dynamics. Proc Natl Acad Sci. 2017:114:E7786-95.

84. Mele M, Ferreira PG, Reverter F, DeLuca DS, Monlong J, Sammeth M, et al. The human transcriptome across tissues and individuals. Science. 2015; 348(6235):660-5.

85. Mao G, Jin H, Wu L. DDX23-Linc00630-HDAC1 axis activates the notch pathway to promote metastasis. Oncotarget. 2017;8(24):38937-49.

86. Milligan MJ, Lipovich L. Pseudogene-derived IncRNAs: emerging regulators of gene expression. Front Genet. 2014;5:476

87. Pink RC, Wicks K, Caley DP, Punch EK, Jacobs L, Francisco Carter DR Pseudogenes: pseudo-functional or key regulators in health and disease? RNA. 2011;17(5):792-8.

88. Jansen R, Batista S, Brooks Al, Tischfield JA, Willemsen G, van Grootheest G, et al. Sex differences in the human peripheral blood transcriptome. BMC Genomics. 2014;15(1):33.

89. Du J, Zhang $X$, Zhou H, Miao $Y$, Han $Y$, Han Q, et al. Alex3 suppresses nonsmall cell lung cancer invasion via AKT/Slug/E-cadherin pathway. Tumour Biol. 2017;39(7):1010428317701441.

90. Sun C, Skaletsky H, Birren B, Devon K, Tang Z, Silber S, et al. An azoospermic man with a de novo point mutation in the Y-chromosomal gene USP9Y. Nat Genet. 1999:23(4):429-32.

91. Luddi A, Margollicci M, Gambera L, Serafini F, Cioni M, De Leo V, et al. Spermatogenesis in a man with complete deletion of USP9Y. N Engl J Med. 2009;360(9):881-5

92. Russo P, Siani A, Miller MA, Karanam S, Esposito T, Gianfrancesco F, et al. Genetic variants of $Y$ chromosome are associated with a protective lipid profile in black men. Arterioscler Thromb Vasc Biol. 2008;28(8):1569-74. 
93. Lopes CA, Prosser SL, Romio L, Hirst RA, O'Callaghan C, Woolf AS, et al. Centriolar satellites are assembly points for proteins implicated in human ciliopathies, including oral-facial-digital syndrome 1. J Cell Sci. 2011;124(Pt 4):600-12.

94. Gregson HC, Van Hooser AA, Ball AR Jr, Brinkley BR, Yokomori K. Localization of human SMC1 protein at kinetochores. Chromosom Res. 2002;10(4):267-77.

95. Bodzioch M, Lapicka-Bodzioch K, Zapala B, Kamysz W, Kiec-Wilk B, Dembinska-Kiec A. Evidence for potential functionality of nuclearly-encoded humanin isoforms. Genomics. 2009;94(4):247-56.

96. Kin T, Sugie K, Hirano M, Goto Y, Nishino I, Ueno S. Humanin expression in skeletal muscles of patients with chronic progressive external ophthalmoplegia. J Hum Genet. 2006;51(6):555-8.

97. Muzumdar RH, Huffman DM, Atzmon G, Buettner C, Cobb LJ, Fishman S, et al. Humanin: a novel central regulator of peripheral insulin action. PLoS One. 2009;4(7):e6334.

98. Hu LT, Zhu BL, Lai YJ, Long Y, Zha JS, Hu XT, et al. HMGCS2 promotes autophagic degradation of the amyloid-beta precursor protein through ketone bodymediated mechanisms. Biochem Biophys Res Commun. 2017:486(2):492-8.

99. Barker DJ, Lampl M, Roseboom T, Winder N. Resource allocation in utero and health in later life. Placenta. 2012;33(Suppl 2):e30-4.

100. Preumont A, Rzem R, Vertommen D, Van Schaftingen E. HDHD1, which is often deleted in X-linked ichthyosis, encodes a pseudouridine-5'phosphatase. Biochem J. 2010;431(2):237-44.

101. Reed MJ, Purohit A, Woo LW, Newman SP, Potter BV. Steroid sulfatase: molecular biology, regulation, and inhibition. Endocr Rev. 2005;26(2):171-202.

102. Lehto M, Mayranpaa MI, Pellinen $T$, Ihalmo $P$, Lehtonen $S$, Kovanen PT, et al. The R-Ras interaction partner ORP3 regulates cell adhesion. J Cell Sci. 2008; 121(Pt 5):695-705.

103. Berx G, van Roy F. Involvement of members of the cadherin superfamily in cancer. Cold Spring Harb Perspect Biol. 2009;1 (6):a003129.

104. Adly N, Alhashem A, Ammari A, Alkuraya FS. Ciliary genes TBC1D32/ C6orf170 and SCLT1 are mutated in patients with OFD type IX. Hum Mutat. 2014;35(1):36-40.

105. Tchaicha JH, Reyes SB, Shin J, Hossain MG, Lang FF, McCarty JH. Glioblastoma angiogenesis and tumor cell invasiveness are differentially regulated by beta8 integrin. Cancer Res. 2011;71(20):6371-81.

106. Xue L, Qiu H, Ma J, Guo M, Li W. ZNF300, a recently identified human transcription factor, activates the human IL-2Rbeta promoter through the overlapping ZNF300/EGR1 binding site. Cell Mol Biol Lett. 2010;15(4):530-40.

107. Akutagawa O, Nishi H, Kyo S, Higuma C, Inoue M, Isaka K. Early growth response-1 mediates up-regulation of telomerase in placenta. Placenta. 2007;28(8-9):920-7.

108. ThomsonAM B, Hytten FE. The assessment of fetal growth. J Obstet Gynaecol Br Commonw. 1968;75:903-16.

109. Hammill PWDT, Johnson CL, Reed RB, Roche AF. NCHS growth charts. Natl Vital Stat Rep. 1976;25(3):1-22.

110. Lubchenco LO, Hansman C, Dressler M, Boyd E. Intrauterine growth as estimated from Liveborn birth-weight data at 24 to 42 weeks of gestation. Pediatrics. 1963:32:793-800

111. Bouwland-Both MI, Steegers EA, Lindemans J, Russcher H, Hofman A, Geurts-Moespot AJ, et al. Maternal soluble fms-like tyrosine kinase-1, placental growth factor, plasminogen activator inhibitor-2, and folate concentrations and early fetal size: the generation R study. Am J Obstet Gynecol. 2013;209(2):121. e1-11

112. Staff ACHN, Braekke K, Hyer M, Hoover RN, Troisi R. Maternal, gestational and neonatal characteristics and maternal angiogenic factors in normotensive pregnancies. Eur J Obstet Gynecol Reprod Biol. 2009;143(1):29-33.

113. Sundrani DKV, Pisal H, Mehendale S, Wagh G, Joshi A, et al. Gestation dependant changes in angiogenic factors and their associations with fetal growth measures in normotensive pregnancy. PLoS One. 2013;8(1):e54153.

114. Mou Z, Tapper AR, Gardner PD. The armadillo repeat-containing protein, ARMCX3, physically and functionally interacts with the developmental regulatory factor Sox10. J Biol Chem. 2009;284(20):13629-40.

115. Wang B, Elledge SJ. Ubc13/Rnf8 ubiquitin ligases control foci formation of the Rap80/Abraxas/Brca1/Brcc36 complex in response to DNA damage. Proc Natl Acad Sci U S A. 2007;104(52):20759-63.

116. Tian D, Sun S, Lee JT. The long noncoding RNA, Jpx, is a molecular switch for X chromosome inactivation. Cell. 2010:143(3):390-403.

117. Buggy JJ, Sideris ML, Mak P, Lorimer DD, Mclntosh B, Clark JM. Cloning and characterization of a novel human histone deacetylase, HDAC8. Biochem J. 2000:350(Pt 1):199-205.
118. Kitagawa R, Bakkenist CJ, McKinnon PJ, Kastan MB. Phosphorylation of SMC1 is a critical downstream event in the ATM-NBS1-BRCA1 pathway. Genes Dev. 2004;18(12):1423-38.

119. Alory C, Balch WE. Organization of the Rab-GDI/CHM superfamily: the functional basis for choroideremia disease. Traffic (Copenhagen, Denmark). 2001;2(8):532-43.

120. Brandl K, Tomisato W, Li X, Neppl C, Pirie E, Falk W, et al. Yip1 domain family, member 6 (Yipf6) mutation induces spontaneous intestinal inflammation in mice. Proc Natl Acad Sci U S A. 2012;109(31):12650-5.

121. Jang SB, Kim YG, Cho YS, Suh PG, Kim KH, Oh BH. Crystal structure of SEDL and its implications for a genetic disease spondyloepiphyseal dysplasia tarda. J Biol Chem. 2002;277(51):49863-9.

122. Wang $Y$, Xia H, Zhuang Z, Miao L, Chen X, Cai H. Axl-altered microRNAs regulate tumorigenicity and gefitinib resistance in lung cancer. Cell Death Dis. 2014:5:e1227.

123. Christov CP, Trivier E, Krude T. Noncoding human Y RNAs are overexpressed in tumours and required for cell proliferation. Br J Cancer. 2008;98(5):981-8.

124. Kelly EE, Giordano F, Horgan CP, Jollivet F, Raposo G, McCaffrey MW. Rab30 is required for the morphological integrity of the Golgi apparatus. Biol Cell. 2012;104(2):84-101.

125. Ikeda M, Hirabayashi S, Fujiwara N, Mori H, Kawata A, lida J, et al. Ras-association domain family protein 6 induces apoptosis via both caspase-dependent and caspase-independent pathways. Exp Cell Res. 2007;313(7):1484-95.

126. Kalscheuer VM, Musante L, Fang C, Hoffmann K, Fuchs C, Carta E, et al. A balanced chromosomal translocation disrupting ARHGEF9 is associated with epilepsy, anxiety, aggression, and mental retardation. Hum Mutat. 2009; 30(1):61-8.

\section{Submit your next manuscript to BioMed Central and we will help you at every step:}

- We accept pre-submission inquiries

- Our selector tool helps you to find the most relevant journal

- We provide round the clock customer support

- Convenient online submission

- Thorough peer review

- Inclusion in PubMed and all major indexing services

- Maximum visibility for your research

Submit your manuscript at www.biomedcentral.com/submit
) Biomed Central 